\title{
A model of protein interactions for regulating plant stem cells
}

\author{
Jérémy Gruel $^{1 *}$, Julia Deichmann ${ }^{1}$, Benoit Landrein ${ }^{1}$, Thomas Hitchcock ${ }^{1}$, Henrik Jönsson ${ }^{1,2,3}$
}

1 Sainsbury Laboratory, University of Cambridge, Bateman Street, Cambridge CB2 1LR, UK. 2 Department of Applied Mathematics and Theoretical Physics, University of Cambridge, Cambridge CB3 0DZ, UK. 3 Computational Biology and Biological Physics, Lund University, 22362 Lund, Sweden.

* Correspondance: jeremy.gruel@slcu.cam.ac.uk

\begin{abstract}
The plant shoot apical meristem holds a stem cell niche from which all aerial organs originate. Using a computational approach we show that a mixture of monomers and heterodimers of the transcription factors WUSCHEL and HAIRY MERISTEM is sufficient to pattern the stem cell niche, and predict that immobile heterodimers form a regulatory 'pocket' surrounding the stem cells. The model achieves to reproduce an array of perturbations, including mutants and tissue size modifications. We also show its ability to reproduce the recently observed dynamical shift of the stem cell niche during the development of an axillary meristem. The work integrates recent experimental results to answer the longstanding question of how the asymmetry of expression between the stem cell marker CLAVATA3 and its activator WUSCHEL is achieved, and recent findings of plasticity in the system.
\end{abstract}

\section{Introduction}

The shoot apical meristem (SAM) is a dome shaped tissue located at the tip of the shoot. The stem cell niche it harbours is at the origin of all the aerial plant organs, making it a critical regulator of plant development [1] It remains active over the lifespan of the plant, continuously providing new cells for developing organs while maintaining its shape and specific gene expression domains stable; a feat enabled by a tight homeostatic control. 
This control is largely dependent on the CLAVATA/WUSCHEL (CLV/WUS) negative feedback loop 2]. A dialogue between the apex of the SAM -the stem cell domain- and its centre is carried out by the transcription factor WUS and the CLAVATA signalling system. WUS is specifically expressed in a central domain; diffusing, it promotes stem cell fate at the tip of the SAM and represses differentiation at its periphery [3] 6 . The 10 CLV3 peptide is expressed in the stem cell domain, and is activated by WUS. It diffuses towards the inner ${ }_{11}$ layers of the meristem where, upon binding the CLV1 receptors, it represses the expression of WUS, thereby 12 closing the feedback loop 7,8. The antagonism between WUS and CLAVATA signalling is reflected in their ${ }_{13}$ perturbations: wus plants exhibit small or even arrested meristems, while clavata plants have massively ${ }_{14}$ enlarged meristems associated with increased organ counts.

The expression of WUS is promoted by cytokinin, making the plant hormone a major factor controlling the SAM homeostasis 9 12]. The expression domains of the enzymes catalysing the synthesis of the active hormone and its receptors stress the importance of the dichotomy between the external cell layers and the inner tissue of the meristem, which can explain the scaling of the SAM domains with its size [13].

The current spatial descriptions of $C L V 3$ regulation, and by extension, of the activation of stem cells, 20 suggest a co-activation by $W U S$ and either an apical [14] or epidermal [5, 6, 13, 15, 16 hypothetical signal, ${ }^{21}$ to generate an asymmetry between the stem cell domain and its main activator WUS. When it comes ${ }^{22}$ to the CLV3 expression domain, the triple hairy meristem (ham) mutant displays a particularly puzzling ${ }^{23}$ phenotype 17. In this mutant, $C L V 3$ is expressed in the centre of the meristem, overlapping with the ${ }_{24}$ expression domain of WUS; the apical or epidermal activation of CLV3 seem difficult to conciliate with this ${ }_{25}^{25}$ observation.

The HAM transcription factors were recently shown to dimerize with $W U S$, with which they share multiple transcriptional targets. HAM1 and HAM2 were also shown to be expressed mostly within the inner tissue of the meristem [18, in a pattern reminiscent of the cytokinin receptors [13].

In the following, we explore the hypothesis that the HAM-WUS dimer represses the expression of CLV3 away from the WUS domain, inspired by the reported central expression of CLV3 in the meristem of ham plants. We first show experimentally that the expression pattern of HAM1 and HAM2 scales with the size 32 of the meristem while remaining mainly expressed in the inner tissue layers; a pattern consistent with an 33 epidermal repression of the two genes. We show that an activation of CLV3 by WUS monomers together 34 with a repression by HAM-WUS dimers is sufficient to pattern the stem cell domain while explaining the 35 triple ham mutant, both using a two-dimensional representation of the meristem and a 3D tissue template 36 generated from confocal microscopy. The resulting model reproduces the asymmetry between WUS and ${ }_{37}$ 
CLV3 expression domains and multiple experimentally described perturbations of the system. It allows for a plastic stem cell domain location and can provide an explanation for this recently observed developmental phenomena.

38

39

\section{Results}

\section{The expression domains of $H A M 1$ and $H A M 2$ scales with meristem size}

The expression domains of HAM1 and HAM2 were described in [18. In both cases, the gene expression was markedly stronger in the center of the meristem than close to the epidermis. Since it is not known what regulates the $H A M$ expression, we introduced perturbations to get an indication of the regulatory motif. To do so, we grew plants in different conditions, such that their SAM size would vary. Whatever the size of the meristematic tissue, the expression of both HAM1 and HAM2 is weak or null close to the epidermis, and relatively stronger in a large central domain in the rib meristem (Fig. 11). Primordia also appear to have a strong influence on the pattern; HAM1 and HAM2 expression are at their strongest where organs emerge.

The expression pattern of the two genes scales with the meristem size, while always avoiding the outer layers of the central SAM. This is consistent with a repression of $H A M$ expression by a signal originating in the epidermis, as previously suggested for the cytokinin receptors [13], and this hypothesis for HAM regulation will be further explored in the following.

\section{An epidermis driven model explains the expression pattern of the stem cell ${ }_{54}$ domain}

Given the HAM domains spatially overlap with WUS, a WUS-HAM dimer would be expected to have its concentration peak in the WUS domain and would therefore be an excellent candidate to explain the exclusion of $C L V 3$ from wild type (WT) meristems, as opposed to ham meristems. However, explaining the apical expression of $C L V 3$ with a combination of two factors expressed directly below is less straightforward, and to explore the hypothesis that HAMs together with WUS, via their physical interaction, are able explain the patterning of the stem cell niche in the SAM, we developed a differential equation model for the spatial expression domains.

In the model (Fig. 2A), WUS expression is regulated by two epidermis-originating morphogens; cytokinin acts as an activator while a second signal abstracting the repression of cyotokinin receptors from the L1 ${ }_{64}$ acts as an inhibitor [13. As suggested by the scaling of HAM1 and HAM2 with the SAM size (Fig. 1), 65 

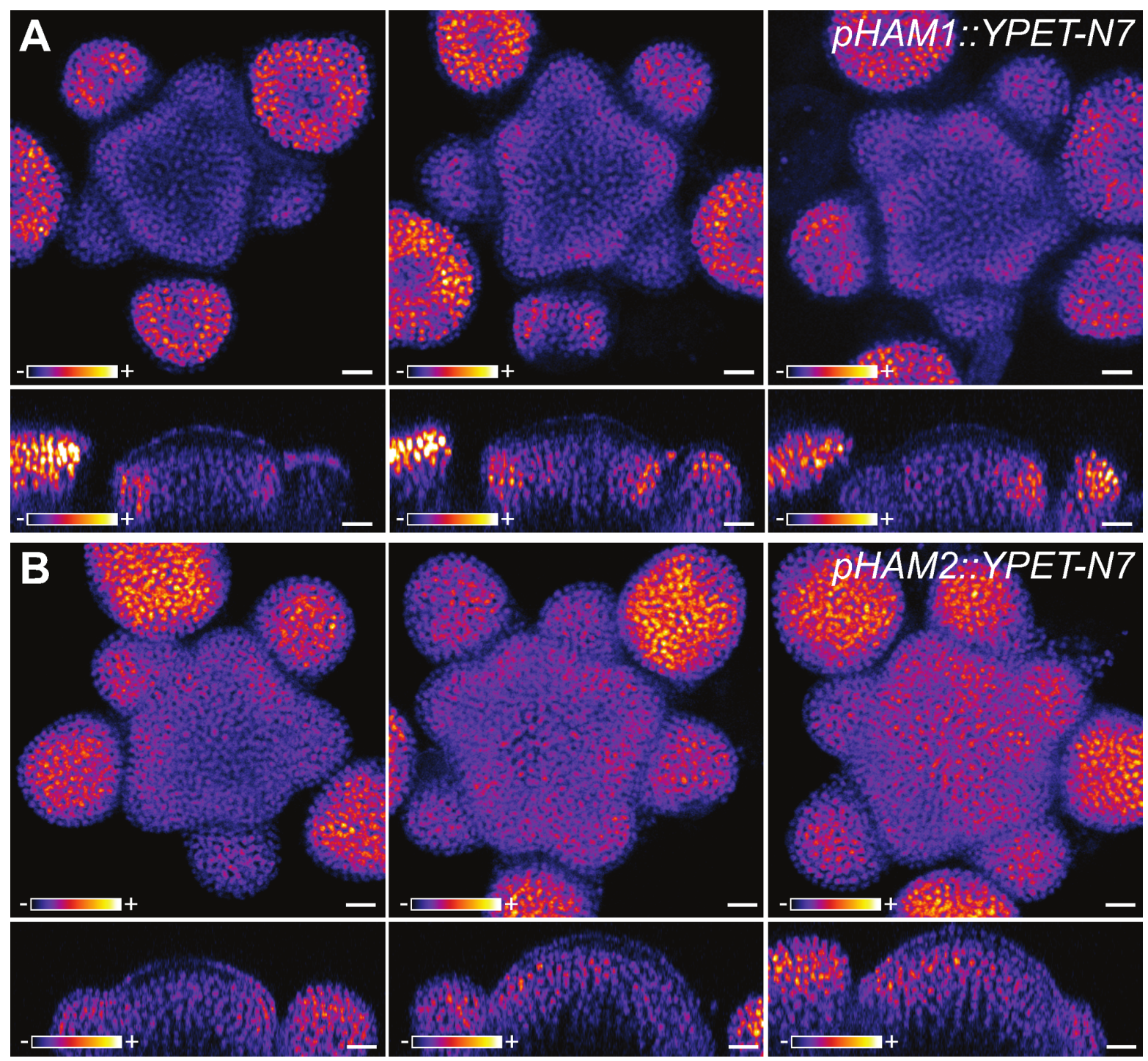

Fig 1: Expression of $p H A M 1:: Y P E T-N 7$ (A) and pHAM2::YPET-N7 (B) in representative meristems of different sizes. Meristems were obtained by growing plants on soil, on soil supplied with fertilizer, or on a mixture of soil and sand, leading to meristems of various sizes. Top images show z-projections (sum slices) and bottom images show sections through the center of the inflorescence meristem. YPET signal is represented using the Fire lookup table of ImageJ. Scale bar: $10 \mu \mathrm{m}$.

a third epidermis originating signal represses the expression of $H A M s$ and $H A M s$, due to their functional redundancy [17, are considered as a single entity. WUS monomers can dimerize with HAM monomers, proteins undergo passive diffusion-like transport (between cells) in the tissue [5, 19. Finally, WUS monomers activate the expression of $C L V 3$ while WUS-HAM heterodimers repress the expression, and CLV3 peptides can move in the tissue and repress the expression of WUS. 

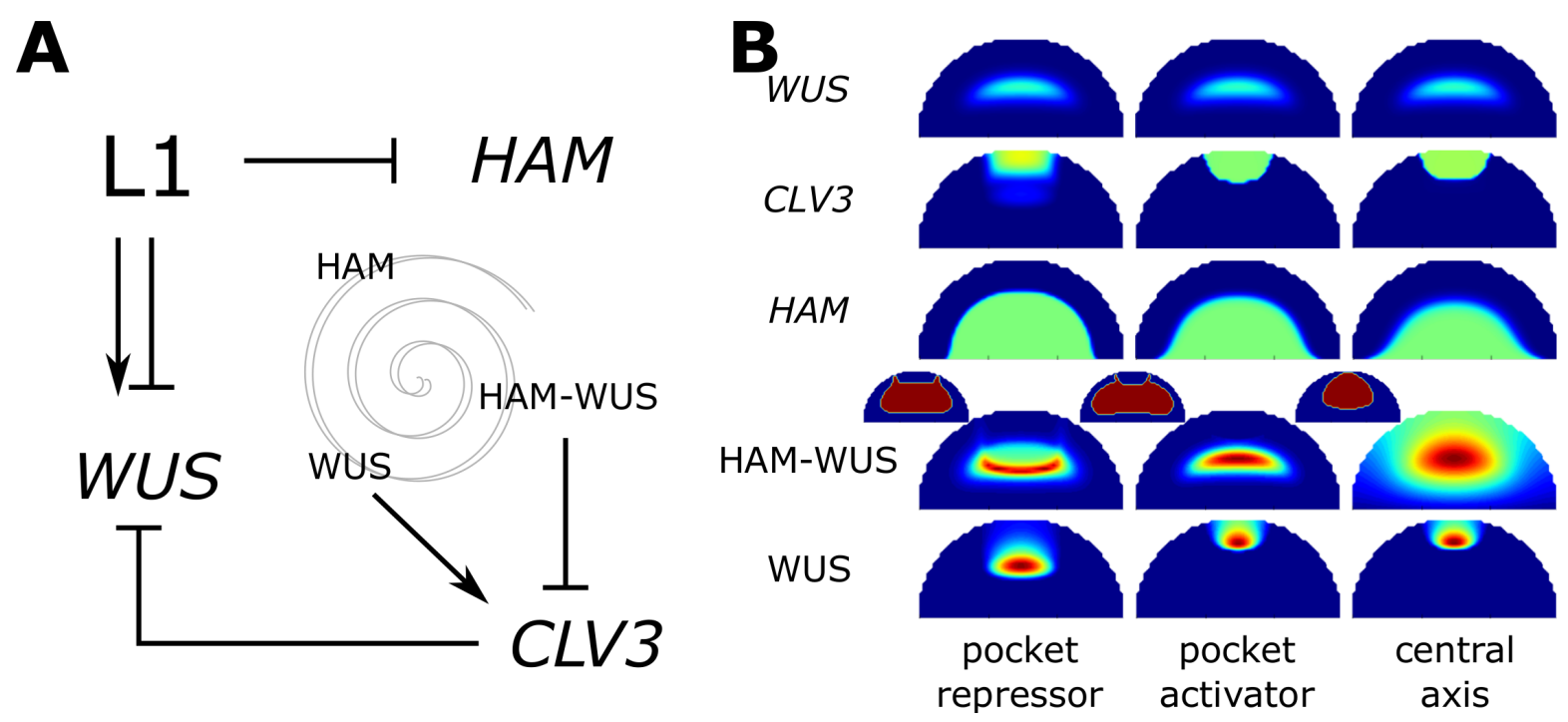

Fig 2: An epidermis controlled model can pattern the stem cell niche. A) Schematic of the model: the epidermis controls WUS expression with an incoherent feed-forward motif, it also represses the expression of HAM. HAM and WUS monomers can heterodimerize; WUS monomer induces the expression of $C L V 3$ while the heterodimer represses it. Finally the CLV3 peptide represses the expression of WUS. B) Parameter optimisations result in three possible behaviours. The panel presents the expression of WUS, CLV3 and $H A M$ on a color scale varying from blue (no expression) to green (optimisation target expression) to red (twice the target expression). HAM-WUS dimers and WUS monomers are plotted on a relative color scale varying from blue (minimal concentration) to red (maximal concentration). Additionally the outline of the pocket formed by the HAM-WUS dimers is displayed (smaller meristems), in blue are concentrations below the maximal value found in the epidermis and in red are concentrations above this value.

The SAM geometry is described as a half disk, the curved part of its perimeter representing the epidermis and its flat part the connection with the plant stem. This representation allows for simple deformations to mimic SAM size and shape dynamics upon mutations or growth condition variations. Model parameters are optimised to reproduce manually defined domains approximating experimental expression for WUS, HAM and CLV3 at equilibrium (Materials and methods, Fig. S1).

Notably, the model achieves to reproduce the expression domains of $W U S, H A M$ and $C L V 3$, and multiple successful parameter values are found with the optimisation procedure (Fig. 23 B). Three broadly different behaviours were identified in the resulting parameter sets. In two of them, the HAM-WUS heterodimers organize in a pocket surrounding the stem cell niche (miniature meristems in Fig. 2B). One case shows WUS monomer concentration peaking in the WUS expression domain, the expression domain of CLV3 is in this case thus mostly delimitated by the repressing HAM-WUS heterodimers; this behaviour will be referred to as "pocket repressor" in the following. The second behaviour displaying a pocket of heterodimers sees WUS monomer concentration peaking in the stem cell domain, directly activating CLV3 and the WUS activation 
plays a prominent role in delineating the $C L V 3$ domain; this behaviour is named "pocket activator". Finally, ${ }^{84}$ the last scenario results in the concentration of all actors of the system peaking along the central vertical axis 85 of the meristem and will be referred to as "central axis". Similarly to "pocket activator", this last case sees 86 WUS monomers peaking in the $C L V 3$ domain. The three categories can be separated in planar projections of ${ }^{87}$ the parameter space (Fig. S2).

As all three alternative behaviours can explain the wild type gene expression domains in the SAM, further analysis is required to assess their biological relevance.

\section{The behaviour of the system hinges on protein differential mobility}

First, we analysed the differences between the defined solutions in terms of predicted protein monomer and dimer mobilities. At equilibrium, the shape of the gradient formed by a diffusing molecule is controlled by the ratio between its diffusion rate and consumption rate. As described in 13 , the epidermis originating morphogenes with a ratio favouring diffusion form a shallow gradient (i.e. cytokinin, acting at long range), while those favouring degradation fall sharply (i.e. the short range AHK repressor). Together these signals form the incoherent feed-forward regulation of WUS generating the adaptive scaling of the expression domain to the size of the meristem.

In the model, WUS and HAM monomers influence the mobility of each other. Indeed, if a WUS monomer is in the presence of sufficient HAM monomers, rather than diffusing, it will likely be recruited to form a dimer. This also applies to HAM monomers in the presence of a large amount of WUS monomers. As the WUS and HAM expression domains are different, the mobility of the monomers is influenced by their location within the meristem. HAM-WUS heterodimers are consumed by degradation and dissociation. The efficiency of those two reactions is not influenced by secondary species, nor is their diffusion rate; the moblility of the dimers does not vary across the tissue. We can achieve a good separation of the classes within the mobility parameter space (6 parameters), showing the relevance of this concept to compare the possible behaviours (Fig. S3).

We analysed the mobility of the two monomers and the dimer across the central axis of the SAM (Fig. 3). 108 For each of the three categories, and as expected, WUS monomers are more mobile close to the apex of the 109 tissue, where relatively little HAM is found, compared to close to the stem, where HAM is expressed. The ${ }_{110}$ opposite holds true for HAM monomers for all three categories as HAM monomers are more mobile close 111 to the stem than they are at the apex. However, the three categories can be differentiated when monomer ${ }_{112}$ mobility is compared to dimer dynamics. In the "central axis" category, dimers are the most mobile of the 113 

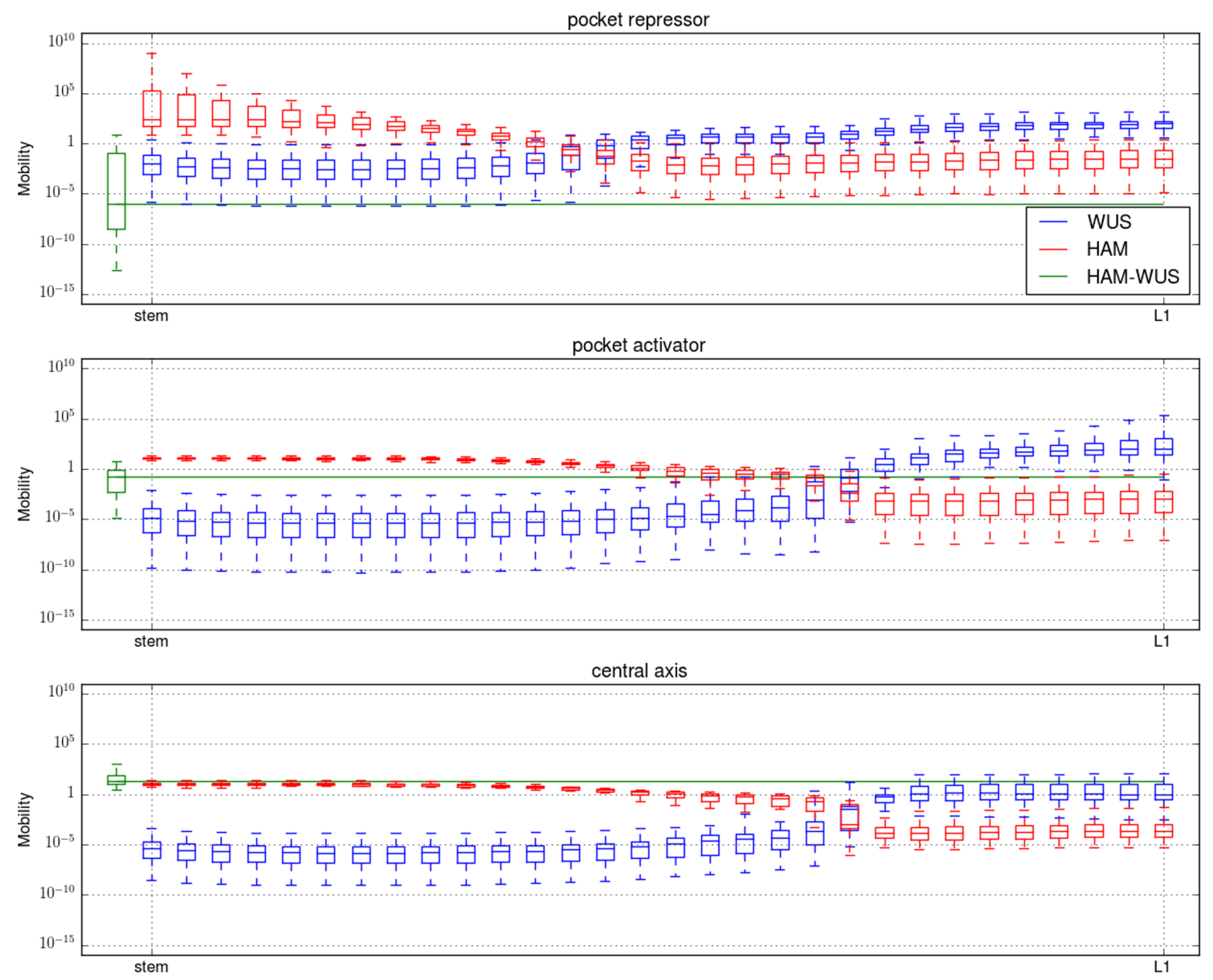

Fig 3: Monomer and dimer mobility. The dimer mobility is computed following $\frac{D}{g+b}$ and monomer mobility following $\frac{D}{g+f[P]}$ with $D$ the diffusion rate of the considered molecule, $g$ its degradation rate, $b$ dimers dissociation rate, $f$ dimers formation rate and $[P]$ the concentration of the monomeric partner of the considered monomer. For all three panels, the $y$ axis is the mobility on a logarithmic scale, the $x$ axis is the central axis of the meristem, with the stem to the left and the apex to the right. For each considered molecule (WUS: red, HAM: green, HAM-WUS: blue), and each position, the mobility value is presented as a boxplot encompassing all optimised parameters belonging to one of the three parameter set categories.

three species. For the "pocket repressor" category, dimers are the least mobile species. For the "pocket ${ }_{114}$ activator" category, the dimer mobility is between the maximum and minimum mobility of both dimers. ${ }_{115}$

It has been suggested that WUS moves between cells via the plasmodesmata, and that the size (e.g. ${ }_{116}$ number of GFP connected to WUS) can be restrictive for the mobility [5] 19. Given this, the fast moving ${ }_{117}$ dimers observed in the "central axis" category would be a less likely scenario. 
bioRxiv preprint doi: https://doi.org/10.1101/237933; this version posted December 21,2017 . The copyright holder for this preprint (which was not certified by peer review) is the author/funder, who has granted bioRxiv a license to display the preprint in perpetuity. It is made available under aCC-BY-NC 4.0 International license.

\section{Perturbations expose behavioural differences between model categories}

Next, we explored various perturbations of the system to expose possible variations in the response of the
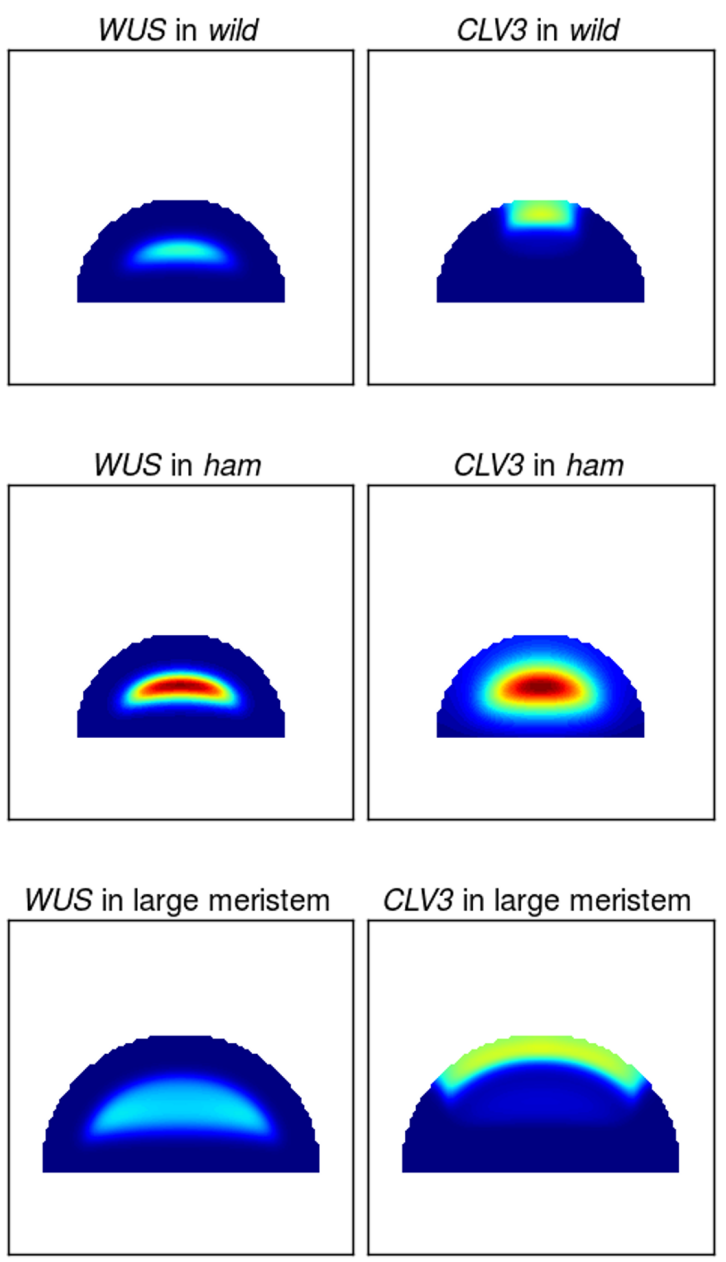
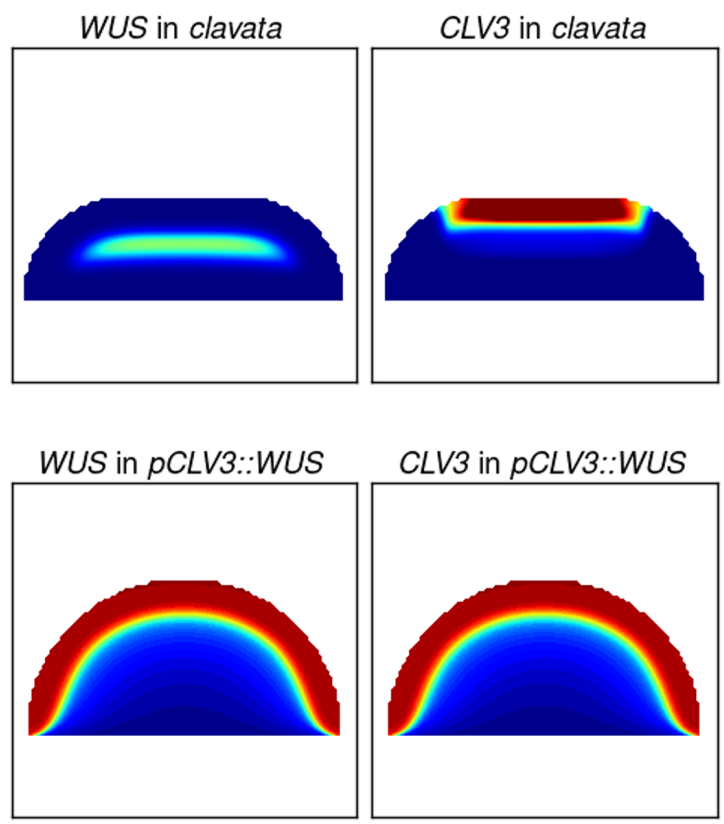

Fig 4: Perturbation examples. Each panel plots either the expression of WUS or CLV3 in various scenarios (wild type, clavata, ham, pCLV3:: WUS and large meristem) using an example parameter set from the "pocket repressor" category and where parameters have been adjusted to represent the mutants (Material and Methods). The colour map for wild type, clavata and large meristems is the same, varying from blue (no expression) to red (twice the wild type target expression for optimisations (green) and any value above). The colour map in the ham and $p C L V 3:: W U S$ scenarios varies between blue and red (minimum and maximum gene expression in the considered settings).

A perequesite for any model aiming at describing the stem cell dynamics of the shoot apical meristem 
is the ability to reproduce the clavata mutants. Abolishing the feedback between $C L V 3$ and WUS yields $\quad{ }^{125}$ fasciated meristems showing a reorganisation of gene expression domains; CLV3 and WUS are expressed in ${ }_{126}$ two band like domains spanning the entirety of the upper cell layers of the expanded tissue 13,20 , (with $\quad{ }_{127}$ CLV3 expression in the 3 to 4 first cell layers and WUS expression from the 3rd cell layer, the genes notably ${ }_{128}$ overlap in the third cell layer).

To investigate the mutant behavior across parameter values for the three categories, the expression of 130 CLV3 and WUS in WT and in a clavata fasciated meristem was analysed along the central axis (Fig. 5). ${ }^{131}$ Little differentiates the three categories, and all display an increase of the expression of both genes. If ${ }_{132}$ analysed in more detail, the spatial overlap of the two gene expression domains is however generally larger in ${ }_{133}$ the "central axis" and "pocket activator" categories (high CLV3 expression through more than half the WUS ${ }_{134}$ domain) than it is for "pocket repressor", where the latter category presents on average a behaviour closer to ${ }_{135}$ observations $[13$.
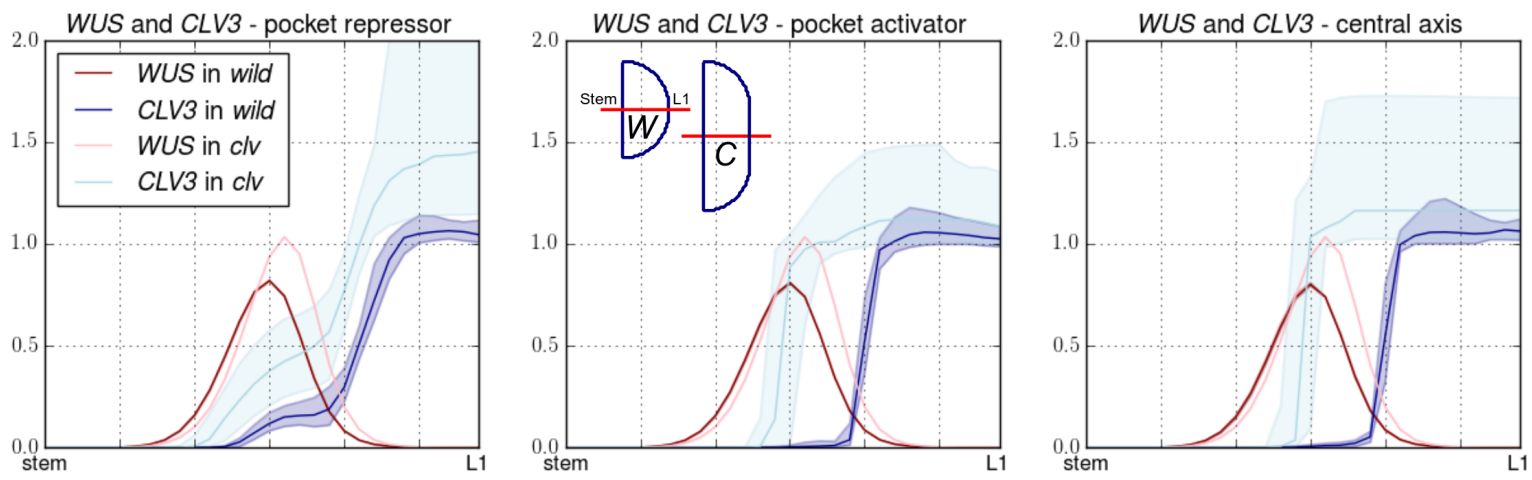

Fig 5: Wild and clavata meristems. The expression of WUS and CLV3 along the central axis of the meristem are plotted for wild type and clavata scenarios. Each plot displays the median expression of the genes along with the first and last quartiles. For comparison, the central panel displays the shape of wild (W) and clavata (C) meristems, their central axis is marked with a red line.

The triple ham mutant phenotype, where $C L V 3$ and WUS expression overlap in a central domain [17], is ${ }_{137}$ another critical feature for the model to achieve. We analysed the relative expression of $C L V 3$ and WUS ${ }_{138}$ along the central axis of the meristem in simulations where the expression of HAM is null (Fig. 6). All ${ }_{139}$ three model categories have parameter values from the optimisation that can achieve a central expression of ${ }_{140}$ CLV3. Within each category, however, the domain is more or less broad and in some cases the domain even ${ }_{141}$ encompasses the whole tissue. The expression of WUS, activated by its epidermal incoherent feed-forward ${ }_{142}$ motif, is always expressed in a central domain. Due to the overlapping expression of $C L V 3$, repression ${ }^{143}$ 
increases and WUS is consistently less expressed compared to wild type. Existing data does not suggest a massive drop of WUS expression in ham mutants, suggesting that either the mutation affects components of the system not modelled in this study (such as CLV3 receptors) or that the CLV3 effect on WUS expression is somehow buffered, as previously suggested $[21$.
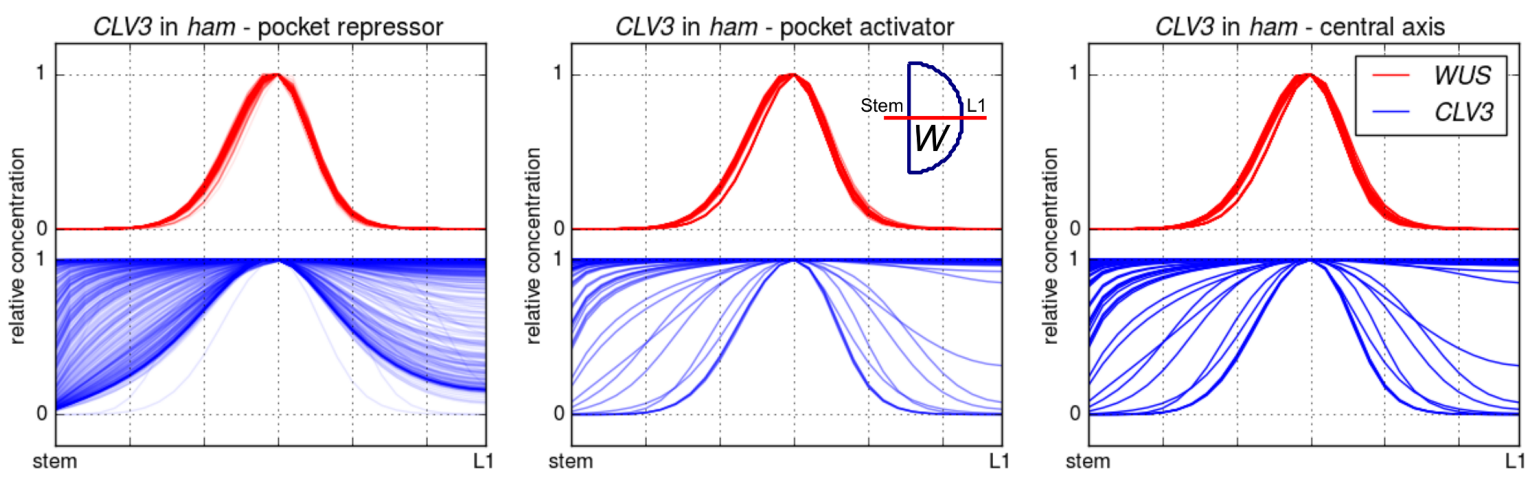

Fig 6: ham meristems. The expression of WUS (red) and CLV3 (blue) along the central axis of the meristem is plotted for the ham scenario. Each line of each plot presents a parameter set and the gene expressions are scaled between 0 and 1 (minimum and maximum expression in the studied parameter set). Simulations were run on the wild type meristem shape. The transparency of the lines is adjusted in each panel for better display.

A third mutant with an interesting and non-trivial phenotype is the $p C L V 3:: W U S$ described in 22 . Plants in which WUS expression is driven by CLV3 promoter exhibit a massively enlarged meristem in ${ }_{149}$ which both $C L V 3$ and $W U S$ are expressed in the three outermost cell layers of the tissue. While the direct ${ }_{150}$ activation of $C L V 3$ by an hypothetical L1 originating morphogen provides a straightforward way of achieving 151 this behaviour 6, 13], it is not obviously the case when WUS and HAM interactions control the activity of ${ }_{152}$ the $C L V 3$ promoter. The characteristic expression domains of $C L V 3$ and $W U S$ in this mutant can also be ${ }_{153}$ displayed along the central axis (Fig. 7). Notably, a large proportion of the "pocket repressor" models achieve ${ }_{154}$ a correct representation of the experimental data, yet many of them fail the test and result in a situation ${ }_{155}$ where both genes are expressed at high levels across the whole tissue. The two other categories display ${ }_{156}$ such flat expression behaviour in a majority of the parameter values, and when they achieve an expression ${ }_{157}$ constrained to the outer cell layers, they do encompass more layers compared to what is seen in experimental ${ }_{158}$ data. Once again, the "pocket repressor" models fit data more closely than the other categories.

Finally, we tested the resilience of the wild type models to an increase of the tissue size (Fig. 8). "Pocket ${ }_{160}$ repressor" models behave more closely to what is seen in experiments in this situation [13], where a large ${ }_{161}$ majority of them maintain correct expression patterns (central WUS domain surmounted by an apical CLV3 ${ }_{162}$ 

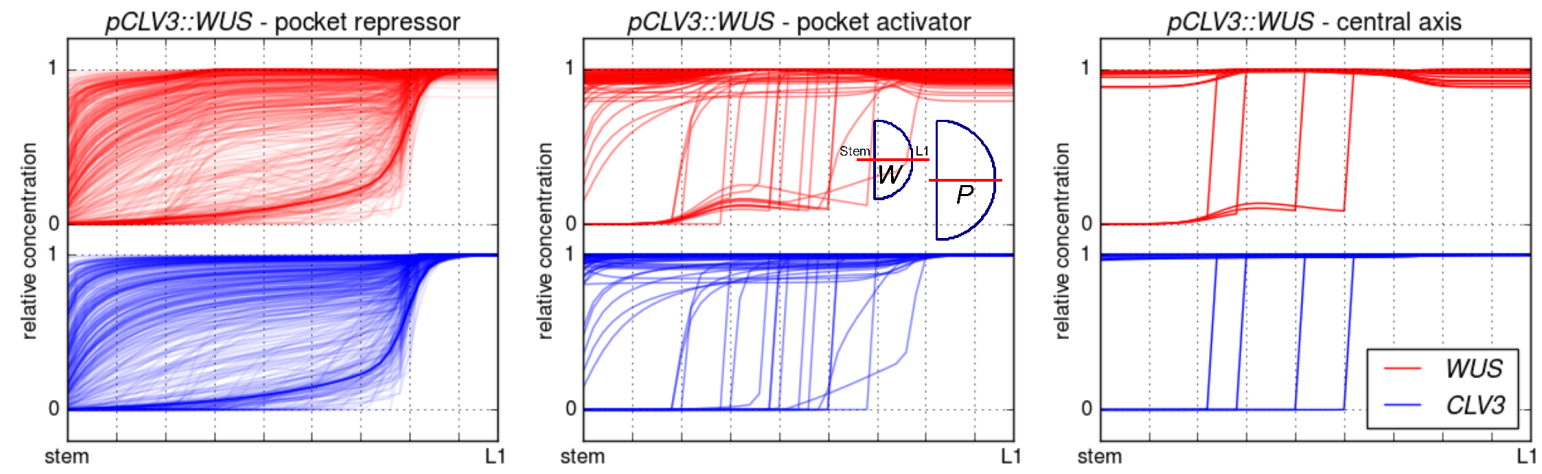

Fig 7: $\boldsymbol{p} \boldsymbol{C L} \boldsymbol{V} 3: \mathbf{W} \boldsymbol{U S}$ meristems. The expression of WUS (red) and CLV3 (blue) along the central axis of the meristems is plotted for the $p C L V 3:: W U S$ scenario. Each line of each plot presents a parameter set and the gene expressions are scaled between 0 and 1 (minimum and maximum expression in the studied parameter set). The transparency of the lines is adjusted in each panel for better display. Simulations were run on the large meristems $(\mathrm{P})$; the geometry is compared to the wild type $(\mathrm{W})$ meristems in the central panel.

domain). In the two other categories the spatial segregation of the expression domains often breaks down, and results in a centrally expressed $C L V 3$ domain.
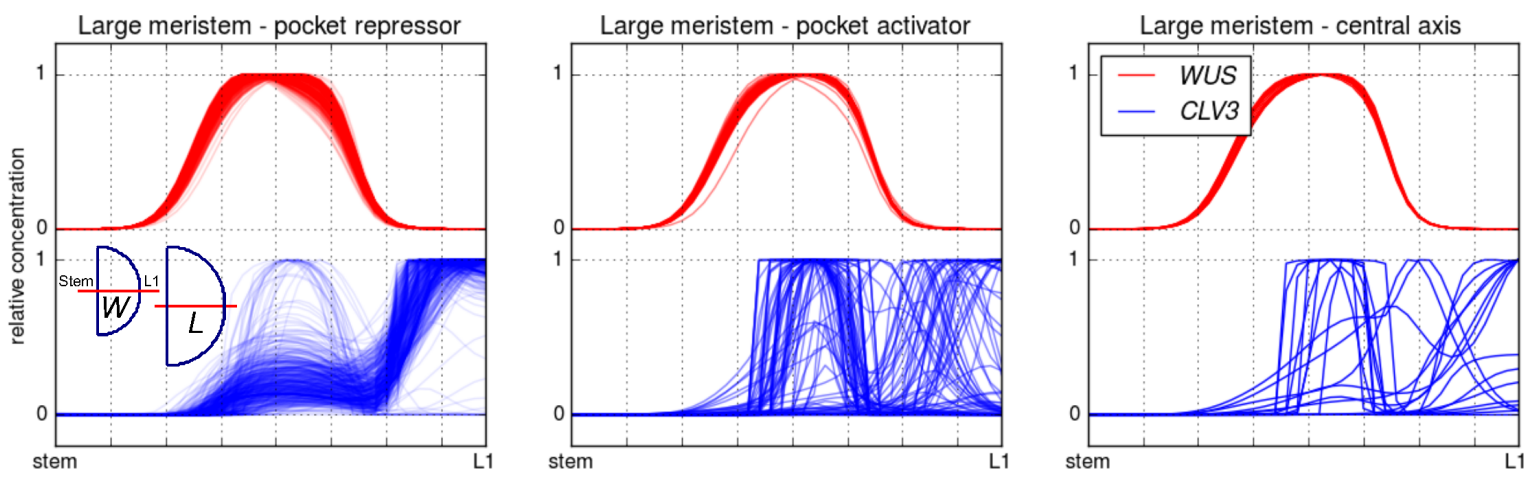

Fig 8: Large meristems. The expression of $W U S$ and $C L V 3$ along the central axis of the meristems is plotted large meristems. Each line of each plot presents a parameter set and the gene expressions are scaled between 0 and 1 (minimum and maximum expression in the studied parameter set). The transparency of the lines is adjusted in each panel for better display. A comparison between large (L) and wild type (W) meristems is presented in the left panel.

The various mutants explored show a variety a behaviours for each of the categories, with the "pocket ${ }_{165}$ repressor" category generally achieving the best results. In order to assess the ability of a single subset of 166 parameters to correctly describe all tested behaviours, we singled out the parameter sets best describing 167 the $p C L V 3::$ WUS mutant (Fig. 9, designated as "pocket repressor +"). When the "pocket repressor +" ${ }_{168}$ parameter sets were tested against the other mutants, they also proved more successful than the rest of the 169 
category ("pocket repressor -"). We further explored the mobility of the monomers and dimers for these two
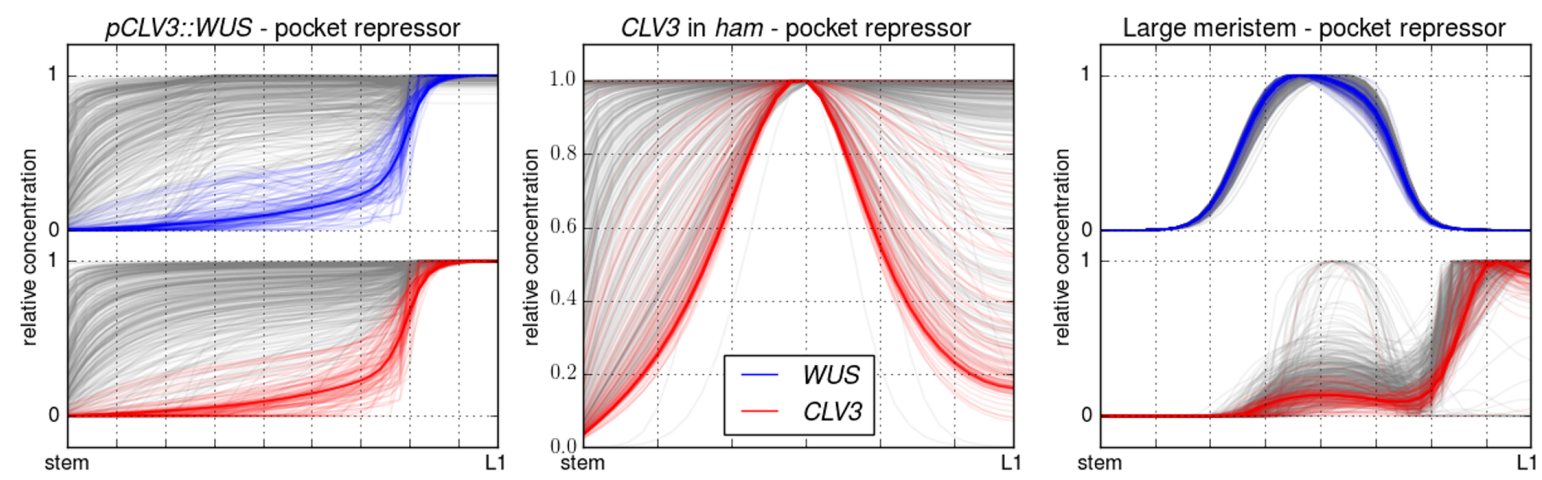

Fig 9: Pocket repressor perturbations exploration. The parameter sets achieving the best description of the $p C L V 3:: W U S$ mutant are selected and coloured (pocket repressor + ), leaving the rest in grey (pocket repressor -). The two sub-categories are mapped on the other two perturbations, following the same colour scheme.
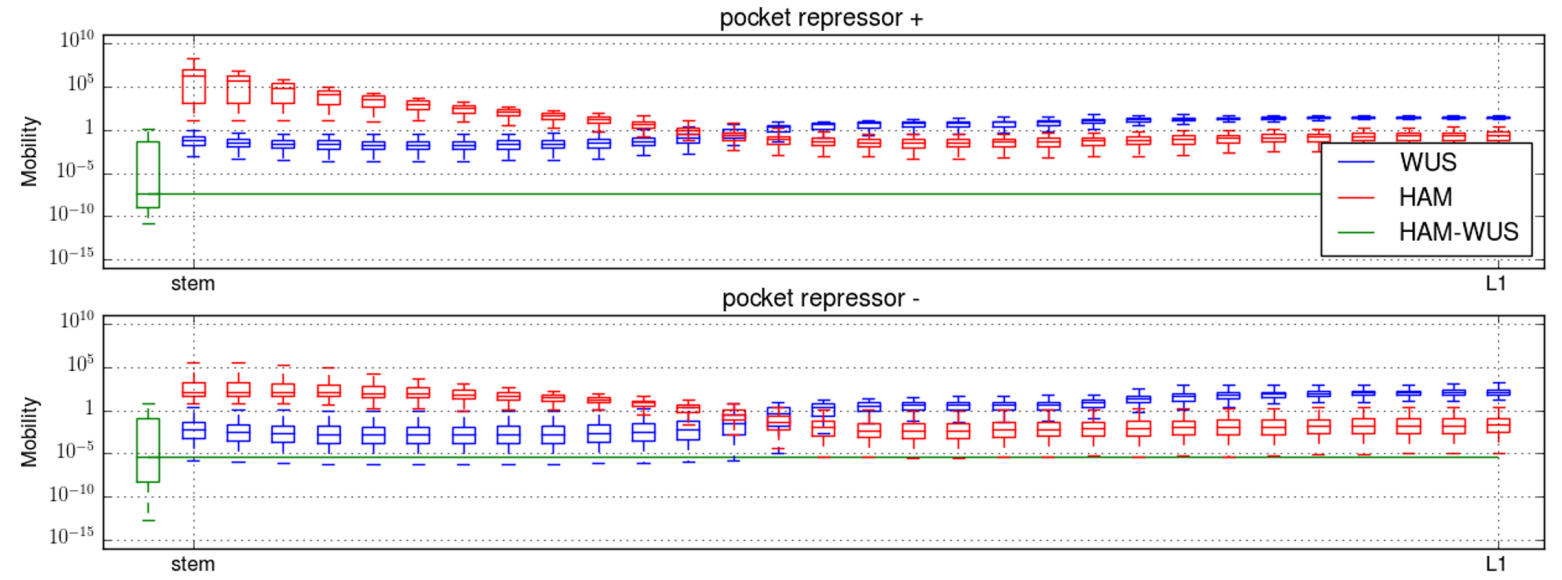

Fig 10: Monomer and dimer mobility in pocket repressor + and -. The dimer mobility is computed following $\frac{D}{g+b}$ and monomer mobility following $\frac{D}{g+f[P]}$ with $D$ the diffusion rate of the considered molecule, $g$ its degradation rate, $b$ dimers dissociation rate, $f$ dimers formation rate and $[P]$ the concentration of the monomeric partner of the considered monomer. For all three panels, the $y$ axis is the mobility on a logarithmic scale, the $x$ axis is the central axis of the meristem, with the stem to the left and the apex to the right. For each considered molecule (WUS: red, HAM: green, HAM-WUS: blue), and each position, the mobility value is presented as a boxplot encompassing all optimised parameters belonging to one of the three parameter set categories. 
The position of $C L V 3$ shifts as HAM concentration varies.

Recent studies have stressed the dynamics of $C L V 3$ expression, providing interesting test cases for the HAM 175 based model. Notably, during the development of axillary meristems in the leaf axils, a WUS domain is 176 established centrally in the tissue followed by an overlapping $C L V 3$ domain. As the meristem matures, the ${ }_{177}$ CLV3 domain gradually shifts from its central position to the tip of the organ 23]. The WUS-HAM model ${ }_{178}$ can reproduce this shifting behaviour of $C L V 3$ expression by manipulating HAM production (Fig. 11). This ${ }_{179}$ suggests that during the development of axillary meristem the WUS and CLV3 domains are first established, ${ }^{180}$ followed by a gradual expression of HAM proteins. As the HAMs reach their normal expression, the $C L V 3$ domain shifts from the center of the organ to its tip.

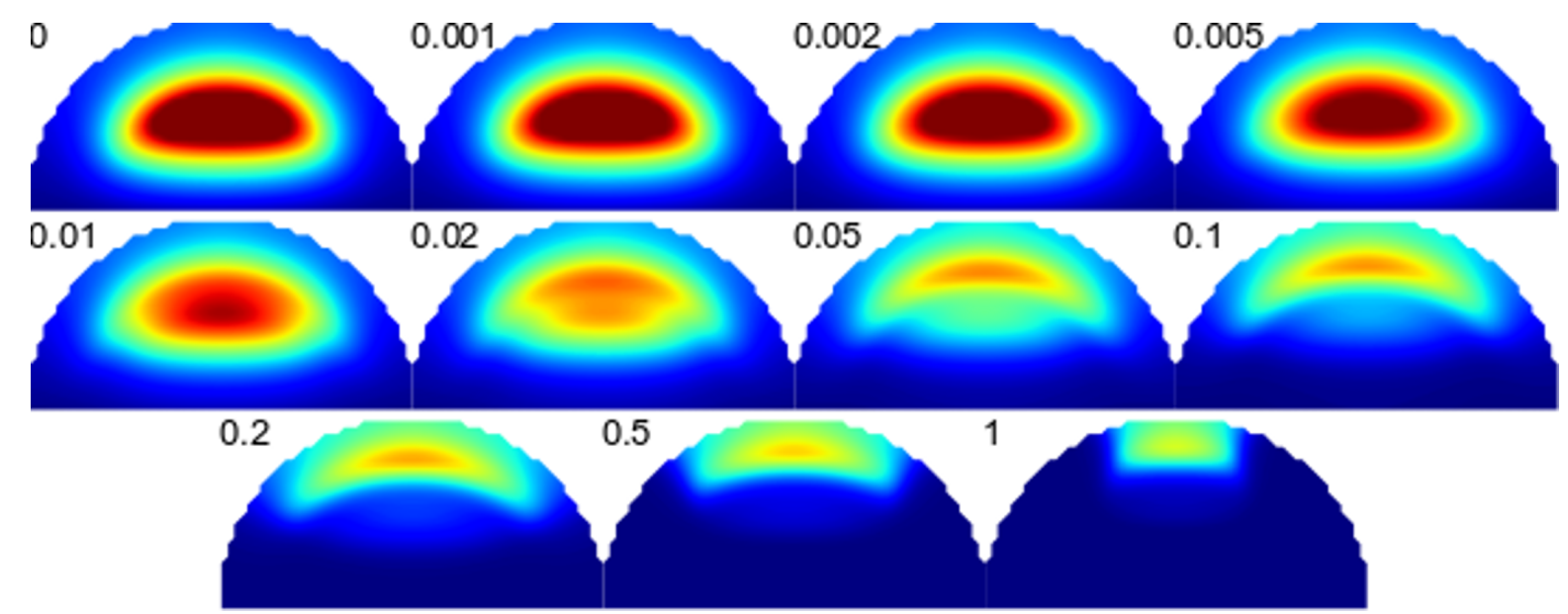

Fig 11: $\boldsymbol{C L} \boldsymbol{V} 3$ domain shift. For the example parameter set, the production of the HAM genes varies from 0 to 1, the factor is indicated along each panel. The color scale for $C L V 3$ expression varies from blue (null) to dark red (twice the wild type expression and above).

This observation also relates to recent observations made in plants with an altered $C L V 3$ promoter. As ${ }_{183}$ WUS binding sites are deleted, the expression of $C L V 3$ can be shifted to different locations between the apex ${ }_{184}$ and the the center of the meristem [24]. The authors discuss multiple hypothesis to explain the phenomenon, ${ }^{185}$ involving either WUS and WUS homodimers and/or possibly the involvement of the HAM proteins. Using ${ }_{186}$ an optimisation approach similar to the one described for the WUS-HAM model (Materials and methods), ${ }^{187}$ we were not able to achieve a correct patterning of $C L V 3$ in models exclusively using WUS monomers and ${ }_{188}$ homodimers when the number of dimensions of the geometrical template is higher than one (Fig. S4). This ${ }_{189}$ leads us to favour the HAM-WUS hypothesis, compared to a WUS-WUS hypothesis as the main regulator of 190 CLV3 expression patterning. 
bioRxiv preprint doi: https://doi.org/10.1101/237933; this version posted December 21, 2017. The copyright holder for this preprint (which was not certified by peer review) is the author/funder, who has granted bioRxiv a license to display the preprint in perpetuity. It is made available under aCC-BY-NC 4.0 International license.

\section{The model is able to describe gene expression patterns on a realistic $3 \mathrm{D}$ meristem} geometry.

To confirm that a 3D geometry or meristem-specific cell neighbourhood topology does not affect the ability of the model to explain the SAM patterning, we applied the developed optimisation strategy to a 3D cell-segmented meristematic tissue [13] (Materials and methods). The description comprises cell volumes and cell contact surfaces for a meristem and early flower primordia. Optimisations resulted in models that successfully describe the gene expression of WUS, HAM and CLV3 (Fig. 12, confirming that the regulatory network in the model is sufficient to generate the SAM patterns. The size of the tissue makes optimisations more difficult and prevents collecting enough parameter sets for a rigorous exploration of the parameter value space. Still, all seven obtained parameter sets belonged to category "pocket repressor". While this analysis does not refute that it is possible to find parameter sets of the other categories, this is an indication that the "pocket repressor" category might be the best descriptor of existing data.

\section{2} 194 95 196 197 198 199 200 201 202 203

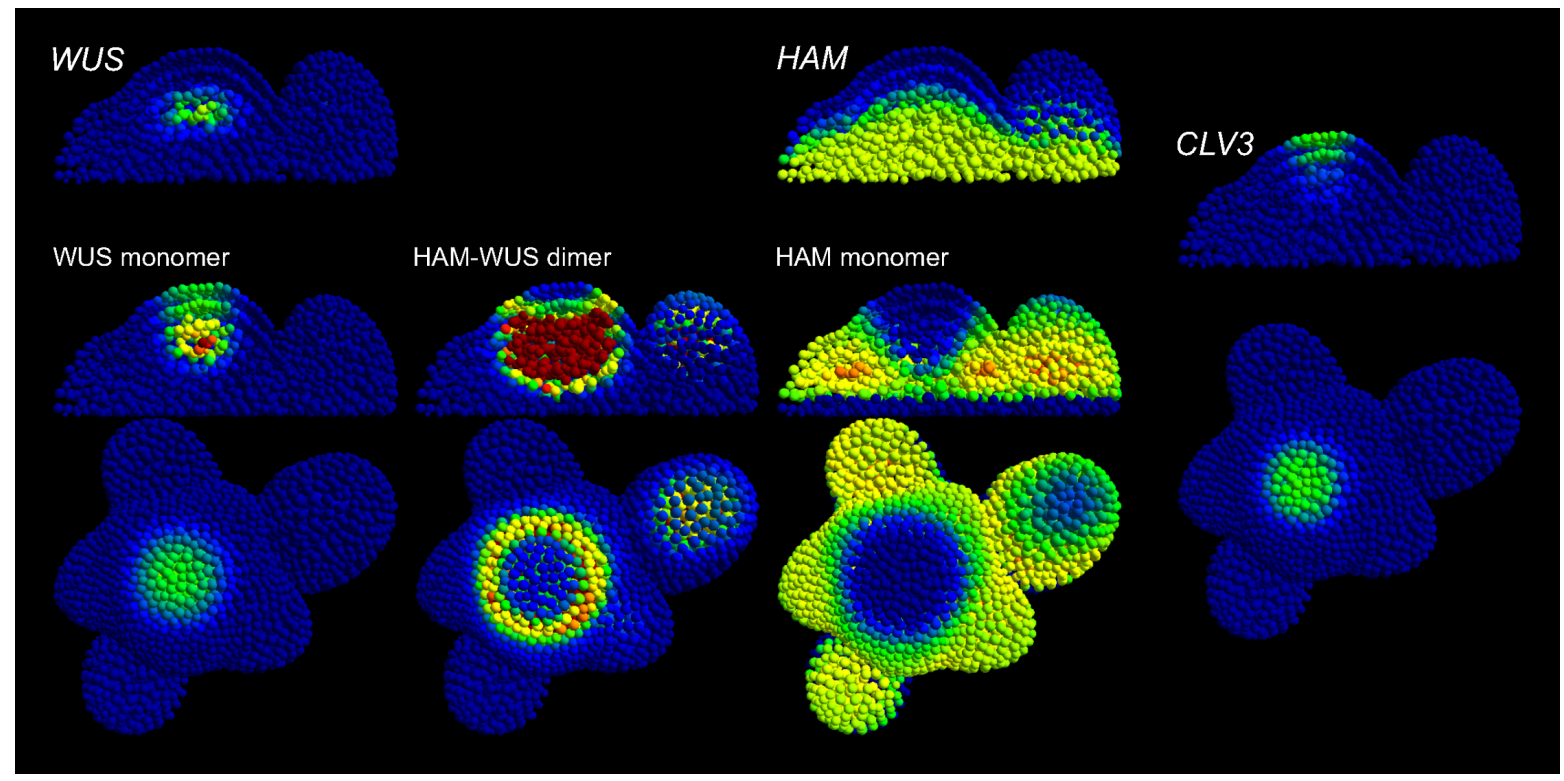

Fig 12: Realistic template. Example behaviour of a parameter set optimised on a realistic template. The gene expression colour map for genes is: blue for no expression, green for optimisation target expression, red for twice the target expression and any value above. The colour map for the monomers varies from blue (minimal concentration) to red (maximal concentration). In order to facilitate the visualisation of the pocket formed by the dimer, any value above the maximum dimer concentration in the L1 is displayed with red; blue indicates a low concentration. 


\section{Discussion}

In this work, we show that two genes ( $W U S$ and $H A M$ ) expressed within the inner layers of the meristematic 205 tissue are sufficient to pattern the apical stem cell niche of A. thaliana. Contrary to previous studies ${ }^{206}$ hypothesising a direct regulation of $C L V 3$ by either apical or epidermal signals, the model, based on recent ${ }^{207}$ experimental data, achieves the plasticity required to explain the wild type and non-apical expression of $C L V 3 \quad 208$ observed in multiple cases: ham [17], mutations of CLV3 promoter [24], lateral buds [23]. This plasticity is 209 achieved through the double negative feedback, where the epidermis represses $H A M$, itself repressing $C L V 3{ }_{210}$ together with WUS, and the adaptive scaling of the $H A M$ expression domain itself to meristem size was 211 confirmed in experiments where plants were grown under various nutrient conditions.

Due to the scarcity of available experimental data, we chose an exploratory strategy allowing, not only to 213 test the potential of the model to describe the stem cell niche, but also to explore various ways of doing so. Out 214 of three identified categories, one exhibits a more biologically probable behaviour. In the "pocket repressor" ${ }^{215}$ category, where the HAM-WUS dimer forms a pocket repressing the expression of CLV3 both WUS and ${ }^{216}$ HAM monomers are more mobile than the dimer they form. In the "pocket repressor + sub-category, where ${ }^{217}$ this diverging behaviour is even more pronounced, the model successfully represents a host of perturbations ${ }_{218}$ of the system, including mutants and tissue size modifications; the other categories fail to consistently achieve 219 such results.

The main feature of the category of models able to reproduce experimental data is the repressing pocket ${ }^{221}$ formed by the heterodimer of WUS and HAM. While the protein mobility fits well with data on WUS, which ${ }^{222}$ have been shown to move between cells via plasmodesmata and where this movement is necessary for correct 223 meristem regulation [5, 19], this has yet to be confirmed for HAM proteins. Immobile HAM proteins would 224 require a different expression pattern, closer to the repressing pocket, presently achieved via protein mobility. ${ }^{225}$

Similarly, while WUS and HAM have been shown to have shared transcriptional targets [18, an alternative ${ }^{226}$ might be that HAM regulation comes solely from depleting part of the meristem from WUS monomers and ${ }_{227}$ hence indirectly inactivating $C L V 3$ expression. This can lead to a similar equilibrium regulation of $C L V 3 \quad 228$ expression, but would more closely resemble the situation of the "pocket activator" than the "pocket repressor" ${ }_{229}$ category of solutions, and hence direct repression by the dimer is predicted by the model. Combining the ${ }^{230}$ "pocket repressor" category with inactivation by WUS depletion would require the intervention of additional 231 species to repress $C L V 3$ from the center of the meristem, such as WUS-WUS homodimers. This would ${ }^{232}$ however affect the ability of the model to reproduce ham mutants, where $C L V 3$ is expressed where the ${ }^{233}$ concentration of all forms of WUS would be highest. 
Finally, in this model and as in [13], the main spatial readouts regulating the expression profile of cells ${ }^{235}$ are various signals originating in the epidermis (such as the long range diffusing cytokinin or the short ${ }_{236}$ range signals repressing cytokinin activity and HAM expression). As such, modifications of the geometry of ${ }^{237}$ the meristematic tissue are directly translated into modifications of the gene expression domains, possibly ${ }^{238}$ explaining how the meristem can adapt the size of its stem cell niche to the size of the host tissue. The ${ }^{239}$ handling of $H A M$ in the model additionally allows it to exhibit a plastic positioning of the stem cell niche, 240 and hence to predict recent experiments displaying such plasticity $17,23,24$.

\section{Materials and methods}

\section{Plant material and imaging conditions}

The $p H A M 1:: Y P E T-N 7$ and $p H A M 2:: Y P E T-N 7$ reporter lines (Ler background) have been described ${ }^{244}$ previously [18. Plants were grown on soil (Levington F2), on a mixture of half soil and half sand, and watered ${ }_{245}$ with or without $1 / 1 / 1$ fertilizer (Vitafeed Standard) and placed in a constant light room $\left(24 \mathrm{~h} \mathrm{light,} 22^{\circ} \mathrm{C}, \quad{ }^{246}\right.$ intensity: $160 \mathrm{\mu mol} \mathrm{m}^{-2} \mathrm{~s}^{-1}$ ) until bolting stage. Imaging was performed as follows: the main inflorescence ${ }_{247}$ meristem was cut, dissected under a binocular stereoscopic microscope to remove all the flowers down to ${ }^{248}$ stage 3 (as defined in 25]) and transferred to a box containing an apex culture medium (ACM) as described ${ }_{249}$ in 26. Meristems were imaged in water using a 20X long-distance water objective mounted on a LSM780 250 confocal microscope (Zeiss, Germany). Z-stacks of $2 \mu \mathrm{m}$ spacing were taken. Z-projections (Sum slices) and ${ }^{251}$ orthogonal sections were performed using the ImageJ software (https://fiji.sc/). ${ }^{252}$

The plant count for every conditions was: ${ }^{253}$ pHAM1::YPET-N7:

- soil: 17 plants

- soil + fertilizer: 11 plants

- soil + sand: 20 plants 


\section{Computational methods}

The following describes the differential equations defining the model, the structure used to represent the ${ }^{263}$ meristem as well as the methods used to find the equilibrium of the system of differential equations and to ${ }_{264}$ optimise model parameters. Methods for the latter are in large following previous work [13]. The section ${ }_{265}$ first focuses on the methodology developed to optimise the two dimensional HAM-WUS model, then we ${ }_{266}$ present the modifications to that methodology implemented to test the WUS-WUS model and to optimise the ${ }^{267}$ HAM-WUS model on the segmented meristematic tissue. Software for all the algorithms described hereafter ${ }^{268}$ and the resulting optimised parameter values are available http://gitlab.com/SLCU/TeamHJ/Jeremy/ham. ${ }^{269}$

\section{D Meristem geometry and topology}

A grid is used to represent the meristem (Fig. S1). Centered on the bottom left corner of the grid, a quarter ${ }^{271}$ of a circle is drawn; if the center of a grid cell is located within the circle the cell will belong to the meristem ${ }^{272}$ representation. 273

The boundaries of the meristem representation are straightforwardly extracted from this representation: ${ }_{274}$

- Cells at the bottom of the grid belong to the sink, connecting the meristem to the stem of the plant. ${ }^{275}$

- Cells on the left row of the grid are at located in the center of the meristem representation. This ${ }_{276}$ boundary is implemented as symmetric, allowing the computations to be limited to half the meristem 277 representation.

- Cells located on the edge of the circle are the cells of the epidermis.

For a total number of cells belonging to the meristem, $n$, the $\operatorname{sink} S$ and the epidermis $L$ are exported as $\quad 280$ size $n$ arrays in which each cell is given a value of 1 if belonging to the said boundary and 0 if not. Similarly, ${ }^{281}$ each variable of the model is stored as an array of size $n$.

The neighbourhood $N$ is exported as a $n \times n$ matrix where $N_{i j}=1$ if cells $i$ and $j$ are neighbours and ${ }^{283}$ $N_{i j}=0$ if not. The diagonal $N_{i i}$ records the amount of neighbours of $i$; cells belonging to the symmetric ${ }^{284}$ boundary have an additional neighbour representing their connection with the abstracted other half of the ${ }_{285}$ meristem. 


\section{Gene expression}

WUS and HAM RNA production is modelled with Hill functions. For a set of $N_{A}$ activators $A_{a}$ and $N_{I}$ inhibitors $I_{b}$, the concentration of a RNA $X$ varies as

$$
\frac{d X}{d t}=V \prod_{A_{a}}^{N_{A}} \frac{A_{a}^{n_{a}}}{A_{a}^{n_{a}}+k_{a}^{n_{a}}} \prod_{I_{b}}^{N_{I}} \frac{k_{b}^{n_{b}}}{I_{b}^{n_{b}}+k_{b}^{n_{b}}}-g X
$$

with $V$, the maximal rate of RNA production. The Hill constants $k$ set the required concentration of activators or inhibitors to switch a gene between its active and inactive states. The Hill coefficients $n$ control the slope of the transition between states. $g_{X}$ is the degradation rate of the RNA.The equilibrium of $X$ is given by

$$
X=\frac{V \prod_{A_{a}}^{N_{A}} \frac{A_{a}^{n_{a}}}{A_{a}^{n_{a}}+k_{a}^{n_{a}}} \prod_{I_{b}}^{N_{I}} \frac{k_{b}^{n_{b}}}{I_{b}^{n_{b}}+k_{b}^{n_{b}}}}{g} .
$$

The production of $C L V 3$ RNA, $C$, is modelled following Shea-Ackers dynamics 27]. Considering a single binding site able to bind either WUS monomers, $w$, as activators or WUS-HAM dimers, $d$, as a inhibitors, the regulation is given by

$$
\frac{d[C]}{d t}=V_{C} \frac{k_{w}[w]}{1+k_{w}[w]+k_{d}[d]}-g_{C}[C],
$$

with $V_{C}$ the maximal rate of transcription, $k_{w}$ and $k_{d}$ the association constants for the WUS monomer and ${ }_{288}$ the WUS-HAM dimer. $g_{C}$ is the degradation rate of the RNA. The equilibrium concentration of CLV3 RNA ${ }_{289}$ is given by

$$
[C]=\frac{V_{C} \frac{k_{w}[w]}{1+k_{w}[w]+k_{d}[d]}}{g_{C}} .
$$

\section{Molecular transport}

Molecular movement between cells is modelled by a passive diffusion-like transport. Such diffusing molecules 292 are produced by a gene expression domain $(W U S, C L V 3, H A M)$ or the L1. This domain is referred to as $P,{ }_{293}$ a vector of cell RNA concentrations for gene expression or a vector of 1 or 0 indicating that a cell belongs to ${ }_{294}$ the $\mathrm{L} 1$ or not. The domain is associated to a production rate $p_{x}$ for molecule species $x$.

The bottom cell layer of the tissue represents the sink, $S$. As for the L1, it is a vector of 1 and 0 . In those ${ }_{296}$ cells, diffusing molecules undergo degradation equal to their diffusion rate $D_{x}$, approximating a continued ${ }_{297}$ flux into the non-modeled tissue below the meristem. Diffusing molecules also undergo a passive degradation ${ }^{298}$ 
of rate $g_{x}$.

For a vector of concentration of a diffusing molecule $x$, we have

$$
\frac{d x}{d t}=p_{x} P_{x}-g_{x} x+D_{x} \Delta x-D_{x} S x,
$$

where $\Delta$ is the Laplace operator; transport in the model is assumed to be passive.

For a cell $i$ with $n_{i}$ neighbours $j$, the diffusion of $x$ follows the discretised version

$$
\frac{d x_{i}}{d t}=D\left(\left(\sum_{j}^{n_{i}} x_{j}\right)-n_{i} x_{i}\right) .
$$

The equilibrium state of the considered molecule is found by solving

$$
p_{x} P_{x}-g_{x} x+D_{x} \Delta x-D_{x} S x=0 .
$$

This is done with the sparse.linalg.spsolve function of the SciPy Python package.

\section{Model}

The model describes the RNA concentration variations of WUS $(W), C L V 3(C)$ and HAM1/HAM2 $(H), \quad 306$ their corresponding proteins and peptides (WUS monomers $(w)$, CLV3 $(c)$, HAM1/HAM2 monomers $(h), \quad 307$ HAM-WUS dimers $(d)$ ) and the three positional signals produced by the epidermis (cytokinin $\left(L_{c}\right)$, the AHK 308 repressor $\left(L_{a}\right)$, the HAM repressor $\left.\left(L_{h}\right)\right)$. Each grid cell of the meristematic representation is described by 309 the following system of ten equations: 


$$
\begin{aligned}
\frac{d[W]}{d t} & =V_{W} \times \frac{\left[L_{c}\right]^{n_{L_{c} W}}}{k_{L_{c} W}^{n_{L_{c} W}}+\left[L_{c}\right]^{n_{L_{c} W}}} \times \frac{k_{L_{a} W}^{n_{L_{a} W}}}{k_{L_{a} W}^{n_{L_{a} W}}+\left[L_{a}\right]^{n_{L_{a} W}}} \times \frac{k_{c}^{n_{c}}}{k_{c}^{n_{c}}+[c]^{n_{c}}}-g_{W}[W] \\
\frac{d[H]}{d t} & =V_{H} \times \frac{k_{L_{H}}^{n_{L_{H}}}}{k_{L_{H}}^{n_{L_{H}}}+\left[L_{H}\right]^{n_{L_{H}}}}-g_{H}[H] \\
\frac{d[C]}{d t} & =V_{C} \frac{k_{w}[w]}{1+k_{w}[w]+k_{d}[d]}-g_{C}[C] \\
\frac{d[w]}{d t} & =p_{w}[W]-g_{w}[w]+D_{w} \Delta[w]-D_{w} S[w]-f[h][w]+b[d] \\
\frac{d[h]}{d t} & =p_{h}[H]-g_{h}[h]+D_{h} \Delta[h]-D_{h} S[h]-f[h][w]+b[d] \\
\frac{d[d]}{d t} & =-g_{d}[d]+D_{d} \Delta[d]-D_{d} S[d]+f[h][w]-b[d] \\
\frac{d[c]}{d t} & =p_{c}[C]-g_{c}[c]+D_{c} \Delta[c]-D_{c} S[c] \\
\frac{d\left[L_{c}\right]}{d t} & =p_{L_{c}} L-g_{L_{c}}\left[L_{c}\right]+D_{L_{c}} \Delta\left[L_{c}\right]-D_{L_{c}} S\left[L_{c}\right] \\
\frac{d\left[L_{a}\right]}{d t} & =p_{L_{a}} L-g_{L_{a}}\left[L_{a}\right]+D_{L_{a}} \Delta\left[L_{a}\right]-D_{L_{a}} S\left[L_{a}\right] \\
\frac{d\left[L_{h}\right]}{d t} & =p_{L_{h}} L-g_{L_{h}}\left[L_{h}\right]+D_{L_{h}} \Delta\left[L_{h}\right]-D_{L_{h}} S\left[L_{h}\right]
\end{aligned}
$$

The geometric template used for optimisation includes 732 cells $(i=732)$, making the description of the $\quad 311$ meristem a total of 7320 equations.

\section{Parameter values}

An multi-step model-specific optimisation strategy was designed to infer the parameter values of the model. 314

The main difference with the strategy presented in 13 is the dimerisation of HAM and WUS that introduces 315 additional non-linearities in the model; this step is solved with Newton's method.

The strategy, detailed afterwards, can be summed up as follows:

1. WUS domain is optimised for an induced clavata phenotype

3. $C L V 3$ and $H A M$ domains are optimised for a wild type phenotype

4. CLV3 and HAM domains are optimised for wild type and CLV3 is optimised for clavata and ham 321 phenotypes 
5. CLV3 peptide is optimised to match step 2

All optimisations are carried out with the L-BFGS algorithm from the SciPy Python package, the 325 parameter values are bounded by $\left[10^{-8}: 10^{8}\right]$. After each step described above, the subset of optimised 326 parameters (see below) are kept as fixed values in the following steps.

In the following, $\operatorname{dif} f(P)$ will refer to computing the equilibrium concentration of a diffusing molecule ${ }_{328}$ across the tissue, given a production domain $P . P$ is a cell vector containing either ones (L1) or zeros (non-L1) ${ }_{329}$ if the production domain is the L1. $P$ is a cell vector of RNA concentrations if it refers to the expression 330 domains of WUS, CLV3 or HAM1/HAM2 (the equilibrium computation is described in the Molecular ${ }_{331}$ transport section). Similarly, $e q\left(\left[A_{1}, \ldots, A_{N_{A}}\right],\left[I_{1}, \ldots, I_{N_{I}}\right]\right)$ will refer to computing the equilibrium of a 332 gene expression regulated by $N_{A}$ activators $A$ and $N_{I}$ inhibitors $I$ (both activators and inhibitors are cell ${ }_{333}$ vectors of diffusing molecule concentrations; the equilibrium computation is described in the Gene expression ${ }_{334}$ section); note that WUS and HAM are computed with Hill functions while CLV3 uses Shea-Ackers. 335

Target expression domains for WUS, CLV3 and HAM1/HAM2 were manually defined on the optimisation 336 template $\left(W_{t}, C_{t}\right.$ and $H_{t}-\mathrm{Fig}$ S5). They are cell vectors containing ones (cells expressing the considered ${ }_{337}$ gene) or zeros (cells not expressing the gene).

1) WUS expression domain The first step optimises the WUS domain for a clavata phenotype; the ${ }_{339}$ only regulators of WUS here are cytokinin and the AHK repressor, both modelled as morphogens produced 340 in the L1.

The equilibrium for the $W U S\left(W_{1}\right)$ domain is computed following:

$$
\begin{aligned}
L_{c} & =\operatorname{diff}(L) \\
L_{a} & =\operatorname{diff}(L) \\
W_{1} & =\operatorname{eq}\left(\left[L_{c}\right],\left[L_{a}\right]\right) .
\end{aligned}
$$

The cost function minimises the difference between the equilibrium WUS domain and an increased target WUS domain $\left(W_{t} \times 1.5\right)$ :

$$
E_{W 1}=\sum_{i}\left(W_{1}-1.5 W_{t}\right)^{2}
$$

where the values for $W_{1}$ and $W_{t}$ are from the individual cells $i$. Parameters $k_{L_{c}}, k_{L_{a}}, p_{c}, D_{c}, g_{c}, p_{a}, D_{a}, g_{a}{ }^{342}$ 
are optimised. As CLV3 is not considered in this step, the equation describing WUS dynamics is reduced to ${ }^{343}$

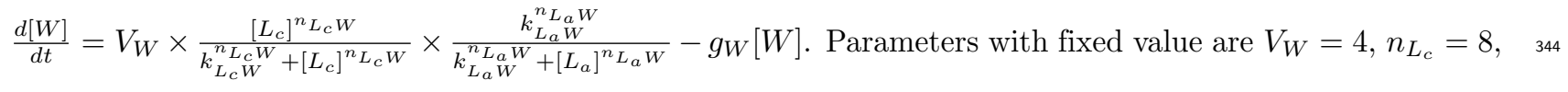
and $n_{L_{a}}=4$.

2) WUS expression domain The second step adds CLV3's effect on WUS; the peptide is produced by the $C L V 3$ target domain. The optimisation minimises the difference between the WUS domain and the WUS target domain. The equilibrium of the wild type WUS domain is given by

$$
\begin{aligned}
c_{t} & =\operatorname{diff}\left(C_{t}\right) \\
W & =W_{1} \times \frac{k_{c}^{n_{c}}}{k_{c}^{n_{c}}+\left[c_{t}\right]^{n_{c}}},
\end{aligned}
$$

and the cost function is

$$
E_{W 2}=\sum_{i}\left(W-W_{t}\right)^{2}
$$

Parameters $k_{c}, p_{c}$ and $D_{c}$ are optimised, and $n_{c}=2$ and $g_{c}=1$ are kept constant (together with 346 parameters optimised in step 1).

3) $\boldsymbol{C L} \boldsymbol{V} 3$ and $\boldsymbol{H A M}$ expression domains This step minimises the difference between $C L V 3$ and 348 HAM1/HAM2 domains and their target domains $C_{t}$ and $H_{t}$. After $W$ and $H$ equilibria are obtained, the 349 equilibrium for the non-linear $w, h, d$ sub-system can be computed using Newton's method leading to the 350 equilibrium for $C$.

With $F(x, W, H)$ the $3 i$ length system containing equations (4), (5) and (6) for all cells, $J(x, W, H)$ its $(3 i)^{2}$ Jacobian matrix and $N(F(x, W, H), J(x, W, H))$ Newton's method applied to find the root of the three-equation system, the equilibrium of $C L V 3$ and $H A M$ is obtained as follows:

$$
\begin{aligned}
L_{H} & =\operatorname{diff}(L) \\
H & =e q\left([],\left[L_{H}\right]\right) \\
w, h, d & =N(F(x, W, H), J(x, W, H)) \\
C & =e q([w],[d]) .
\end{aligned}
$$


The cost function to minimise is given by

$$
E_{C H 1}=\sum_{i}\left(C-C_{t}\right)^{2}+\omega \sum_{i}\left(H-H_{t}\right)^{2}
$$

where we used a weight $\omega=0.08$. Optimised parameters are: $k_{L_{H}}, p_{L_{H}}, D_{L_{H}}, p_{w}, D_{w}, p_{h}, D_{h}, f, D_{d}, V_{C}, \quad 352$ $k_{w}, k_{d}, g_{h}, g_{w}, g_{d}, b$. Fixed parameters are: $V_{H}=1, n_{L_{H}}=4$.

4) $\boldsymbol{C L V} 3$ and $\boldsymbol{H A M}$ expression domains This step uses the previously optimised parameter values as 354 an initial guess to start a second broader optimisation. Here, CLV3 and HAM domains are optimised for 355 wild type and CLV3 is optimised for clavata and ham phenotypes.

With $C_{c}$ and $C_{h}$ the equilibria of $C L V 3$ in clavata and ham phenotypes, the equilibria for the different ${ }_{357}$ genes and conditions are computed by the following procedure:

$$
\begin{aligned}
L_{H} & =\operatorname{diff}(L) \\
H & =e q\left([],\left[L_{H}\right]\right) \\
w, h, d & =N(F(x, W, H), J(x, W, H)) \\
C & =e q([w],[d]), \\
p_{w} & \rightarrow p_{w} \times 1.5 \\
w, h, d & =N(F(x, W, H), J(x, W, H)) \\
C_{c} & =e q([w],[d]), \\
p_{h} & \rightarrow 0 \\
w, h, d & =N(F(x, W, H), J(x, W, H)) \\
C_{h} & =e q([w],[d]) .
\end{aligned}
$$


The cost function is given by:

$$
E_{C H 2}=\sum_{i}\left(C-C_{t}\right)^{2}+\omega_{1} \sum_{i}\left(H-H_{t}\right)^{2}+\omega_{2}\left(\frac{\sum_{i} C_{c}}{\sum_{i} C}-1.5\right)^{2}+\log \sum_{i}\left(W-C_{h}\right)^{2},
$$

with weights $\omega_{1}=0.04$ and $\omega_{2}=0.2$. Parameters $k_{L_{H}}, p_{L_{H}}, D_{L_{H}}, p_{w}, D_{w}, p_{h}, D_{h}, f, D_{d}, V_{C}, k_{w}, k_{d}, g_{h}, \quad 359$ $g_{w}, g_{d}, b$ are re-optimised.

5) CLV3 peptide gradient In this step, the CLV3 peptide gradient produced by the CLV3 domain ${ }_{361}$ optimised in the previous step is fitted to the gradient obtained in step 2) and produced by the target CLV3 362 domain.

$C$ at equilibrium is computed following:

$$
c=\operatorname{diff}(C)
$$

The cost function is given by

$$
E_{c}=\sum_{i}\left(c-c_{t}\right)^{2}
$$

Parameters $p_{c}, D_{c}$ and $g_{c}$ are optimised.

6) Equilibrium of the complete model In the final step, the previously optimised sub-parts of the 366 model are assembled and the equilibrium of the full model is computed. The following algorithm is used: ${ }^{367}$

After multiple optimisation runs, the parameters kept for further analysis are those where $\sum_{i}\left(C-C_{t}\right)^{2}<15 \quad 368$ when the system is in equilibrium.

\section{WUS-WUS model}

In order to test a model in which the combination of WUS monomers and WUS-WUS homodimers would ${ }^{371}$ regulate the expression of $C L V 3$, we used the same core model as previously described.

We removed the equations referring to HAM transcription and HAM monomer (equations 2 and 5). Equation 6, describing the dimer dynamics, was modified to

$$
\frac{d[d]}{d t}=-g_{d}[d]+D_{d} \Delta[d]-D_{d} S[d]+f[w][w]-b[d]
$$




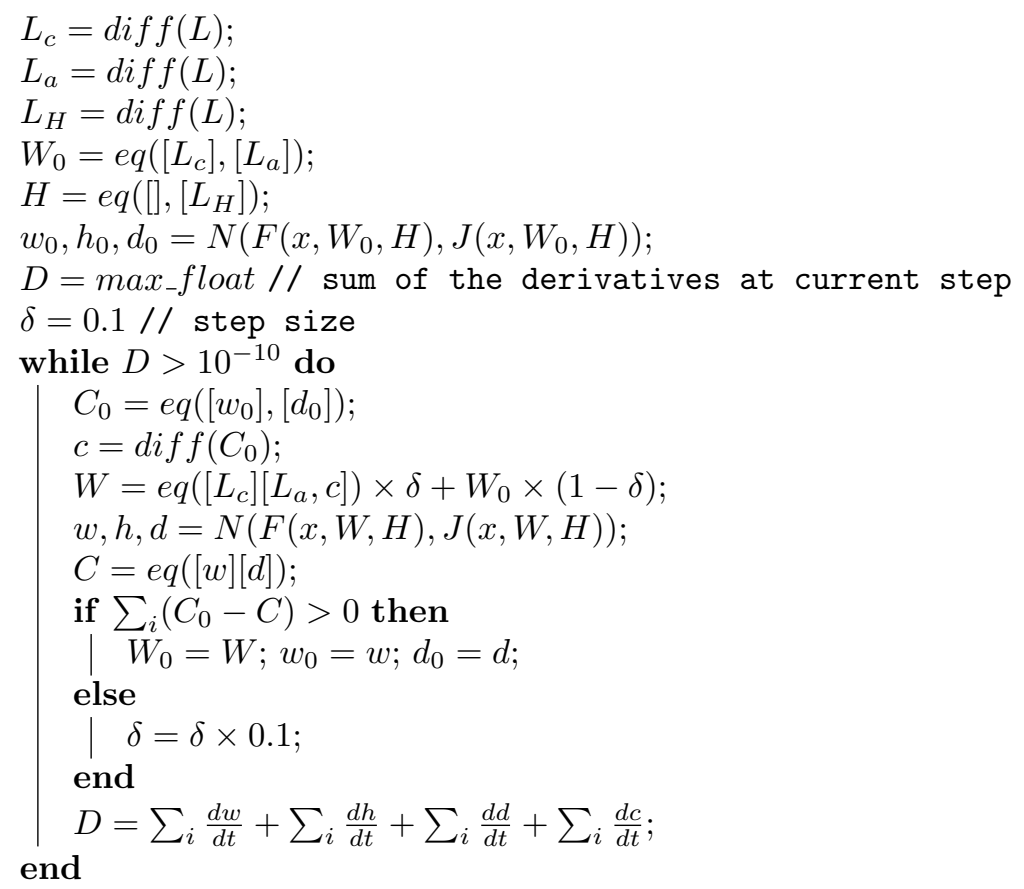

and equation 4, describing WUS monomer dynamics, was modified to

$$
\frac{d[w]}{d t}=p_{w}[W]-g_{w}[w]+D_{w} \Delta[w]-D_{w} S[w]-f[w][w]+b[d]
$$

Finally the components of the optimisation procedure related to HAM expression were left out. Example 373 results obtained with this approach are presented in Fig. S4.

The main difference for the 3D template compared to the two dimensional template is the use of cell volumes and cell contact surfaces as obtained from the segmentation of the confocal imaging of a meristem tissue 13 . The equation controlling the molecular transport of $x$ becomes:

$$
\frac{d x_{i}}{d t}=\frac{D}{V_{i}} \sum_{j}^{n_{j}} C_{i j}\left(x_{j}-x_{i}\right)
$$

for a cell $i$ with $n_{i}$ neighbours $j$, with $V_{i}$ the volume of cell $i$ and $C_{i j}$ the contact surface between cells $i$ and $\quad 376$ $j$. Hill coefficients from the optimisations in [13] were kept: $n_{L_{c} W}=7.25968619416, n_{L_{a} W}=1.99109438845, \quad 377$ $n_{c}=6.66419523049$, and in addition $n_{l H}=6$ was fixed. Other parameter values were found by the same 378 optimisation strategy as used for the 2D templates. 


\section{Acknowledgments}

We thank E. Meyerowitz for providing the HAM1/HAM2 reporter lines and members of the Jönsson and 381 Meyerowitz (Caltech) groups for fruitful discussions. This work was supported by the Gatsby Charitable 382 Foundation via grant GAT3395-PR4.

\section{References}

1. Steeves TA, Sussex IM. Patterns in plant development. Cambridge University Press; 1989.

2. Schoof H, Lenhard M, Haecker A, Mayer KF, Jurgens G, Laux T. The stem cell population of Arabidopsis 386 shoot meristems in maintained by a regulatory loop between the CLAVATA and WUSCHEL genes. $\quad 387$ Cell. 2000;100(6):635-644.

3. Laux T, Mayer KF, Berger J, Jurgens G. The WUSCHEL gene is required for shoot and floral meristem 389 integrity in Arabidopsis. Development. 1996;122(1):87-96.

4. Mayer KF, Schoof H, Haecker A, Lenhard M, Jurgens G, Laux T. Role of WUSCHEL in regulating ${ }^{391}$ stem cell fate in the Arabidopsis shoot meristem. Cell. 1998;95(6):805-815.

5. Yadav RK, Perales M, Gruel J, Girke T, Jönsson H, Reddy GV. WUSCHEL protein movement ${ }^{393}$ mediates stem cell homeostasis in the Arabidopsis shoot apex. Genes Dev. 2011;25:2025-2030. 394

6. Yadav RK, Perales M, Gruel J, Ohno C, Heisler M, Girke T, et al. Plant stem cell maintenance involves 395 direct transcriptional repression of differentiation program. Mol Syst Biol. 2013;9:654. 396

7. Clark SE, Williams RW, Meyerowitz EM. The CLAVATA1 gene encodes a putative receptor kinase ${ }^{397}$ that controls shoot and floral meristem size in Arabidopsis. Cell. 1997;89(4):575-585.

8. Ogawa M, Shinohara H, Sakagami Y, Matsubayashi Y. Arabidopsis CLV3 peptide directly binds CLV1 399 ectodomain. Science. 2008;319(5861):294.

9. Gordon SP, Chickarmane VS, Ohno C, Meyerowitz EM. Multiple feedback loops through cytokinin 401 signaling control stem cell number within the Arabidopsis shoot meristem. Proc Natl Acad Sci USA. 402 2009;106(38):16529-16534. 
10. Bartrina I, Otto E, Strnad M, Werner T, Schmulling T. Cytokinin regulates the activity of reproductive ${ }^{404}$ meristems, flower organ size, ovule formation, and thus seed yield in Arabidopsis thaliana. Plant Cell. 405 $2011 ; 23(1): 69-80$.

11. Chickarmane VS, Gordon SP, Tarr PT, Heisler MG, Meyerowitz EM. Cytokinin signaling as a positional ${ }_{407}$ cue for patterning the apical-basal axis of the growing Arabidopsis shoot meristem. Proc Natl Acad ${ }_{408}$ Sci USA. 2012;109(10):4002-4007.

12. Landrein B, Formosa-Jordan P, Malivert A, Schuster C, Melnyk CW, Yang W, et al. Nitrate ${ }_{410}$ modulates stem cell dynamics by regulating WUSCHEL expression through cytokinins. bioRxiv. ${ }^{411}$ 2017;doi:10.1101/200303.

13. Gruel J, Landrein B, Tarr P, Schuster C, Refahi Y, Sampathkumar A, et al. An epidermis-driven ${ }_{413}$ mechanism positions and scales stem cell niches in plants. Science advances. 2016;2(1):e1500989. 414 doi:10.1126/sciadv.1500989.

14. Hohm T, Zitzler E, Simon R. A dynamic model for stem cell homeostasis and patterning in Arabidopsis ${ }_{416}$ meristems. PLoS ONE. 2010;5(2):e9189.

15. Jönsson H, Shapiro B, Meyerowitz E, Mjolsness E. Signaling in multicellular models of plant development. $\quad 418$ In: Kumar S, Bentley P, editors. On Growth, Form and Computers. London: Academic Press; 2003. p. 419 $156-161$.

16. Adibi M, Yoshida S, Weijers D, Fleck C. Centering the Organizing Center in the Arabidopsis thaliana ${ }_{421}$ Shoot Apical Meristem by a Combination of Cytokinin Signaling and Self-Organization. PLoS ONE. ${ }_{422}$ 2016;11(2):e0147830.

17. Schulze S, Schafer BN, Parizotto EA, Voinnet O, Theres K. LOST MERISTEMS genes regulate cell ${ }_{424}^{424}$ differentiation of central zone descendants in Arabidopsis shoot meristems. Plant J. 2010;64(4):668-678. $\quad 425$

18. Zhou Y, Liu X, Engstrom EM, Nimchuk ZL, Pruneda-Paz JL, Tarr PT, et al. Control of plant stem ${ }_{426}$ cell function by conserved interacting transcriptional regulators. Nature. 2015;517(7534):377-380. ${ }_{427}$

19. Daum G, Medzihradszky A, Suzaki T, Lohmann JU. A mechanistic framework for noncell autonomous ${ }^{428}$ stem cell induction in Arabidopsis. Proc Natl Acad Sci USA. 2014;111(40):14619-14624.

20. Fletcher JC, Meyerowitz EM. Cell signaling within the shoot meristem. Curr Opin Plant Biol. ${ }_{430}$ 2000;3(1):23-30. 
21. Muller R, Borghi L, Kwiatkowska D, Laufs P, Simon R. Dynamic and compensatory responses of ${ }_{432}$ Arabidopsis shoot and floral meristems to CLV3 signaling. Plant Cell. 2006;18(5):1188-1198.

22. Brand U, Grunewald M, Hobe M, Simon R. Regulation of CLV3 expression by two homeobox genes in ${ }^{434}$ Arabidopsis. Plant Physiol. 2002;129(2):565-575.

23. Xin W, Wang Z, Liang Y, Wang Y, Hu Y. Dynamic expression reveals a two-step patterning of WUS ${ }_{436}$ and CLV3 during axillary shoot meristem formation in Arabidopsis. J Plant Physiol. 2017;214:1-6. $\quad$ 437

24. Perales M, Rodriguez K, Snipes S, Yadav RK, Diaz-Mendoza M, Reddy GV. Threshold-dependent tran- ${ }^{438}$ scriptional discrimination underlies stem cell homeostasis. Proc Natl Acad Sci USA. 2016;113(41):E6298- ${ }^{439}$ E6306.

25. Smyth DR, Bowman JL, Meyerowitz EM. Early flower development in Arabidopsis. Plant Cell. ${ }^{441}$ $1990 ; 2(8): 755-767$.

26. Fernandez R, Das P, Mirabet V, Moscardi E, Traas J, Verdeil JL, et al. Imaging plant growth in 4D: ${ }_{443}$ robust tissue reconstruction and lineaging at cell resolution. Nat Methods. 2010;7(7):547-553.

27. Shea MA, Ackers GK. The OR control system of bacteriophage lambda. A physical-chemical model for ${ }_{445}$ gene regulation. J Mol Biol. 1985;181(2):211-230. 


\section{Supporting information}

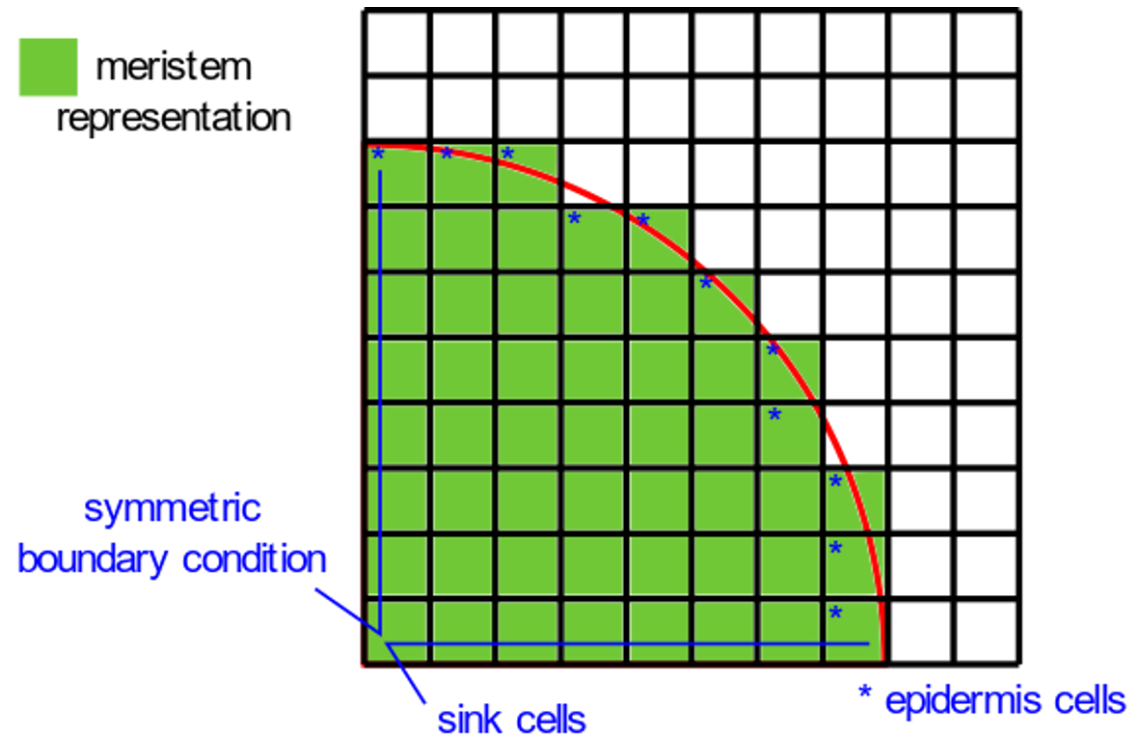

Fig S1: Meristem geometry layout. 


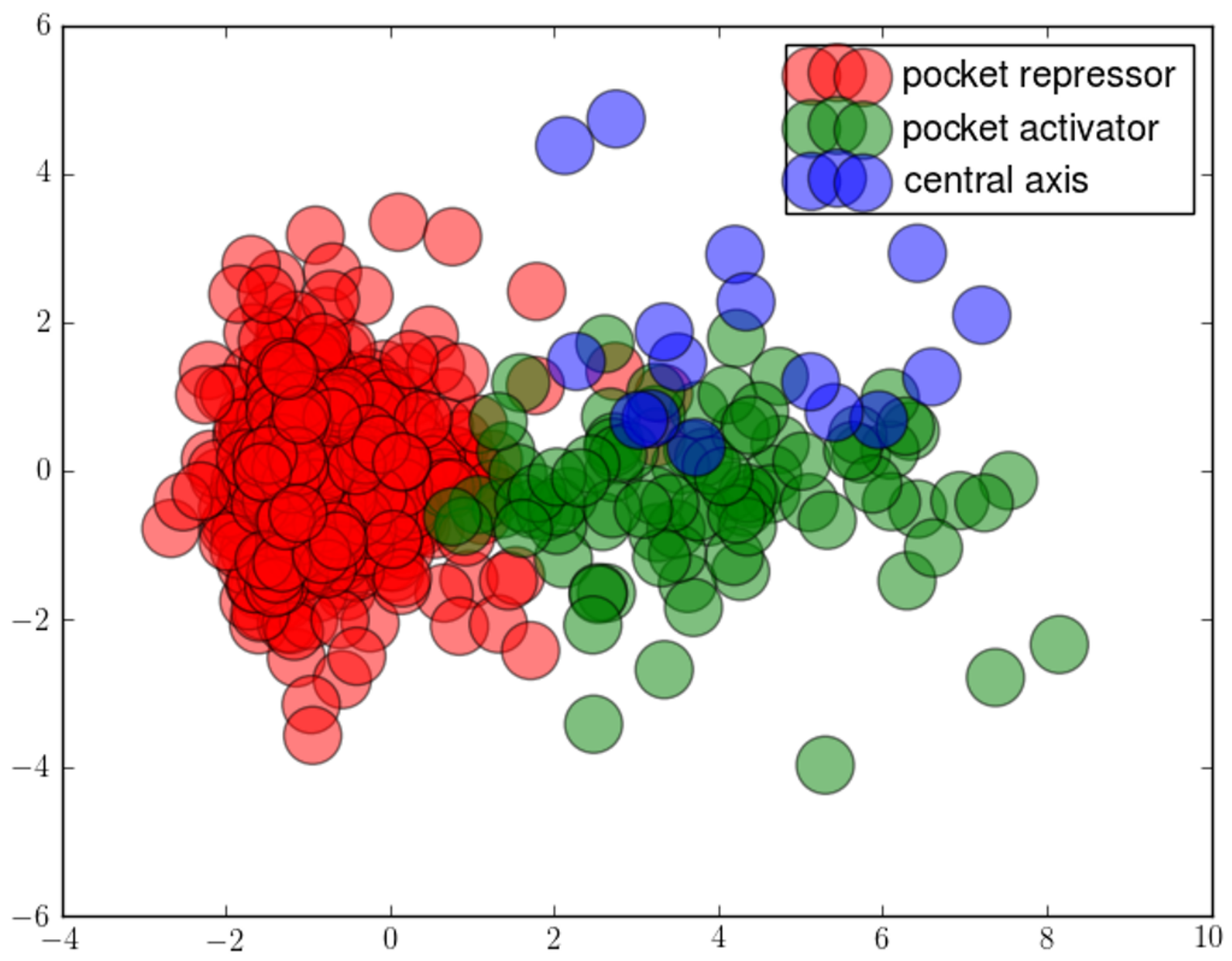

Fig S2: The three categories of behaviour can be separated in the parameter space. The base 10 logarithm of parameter values were centered and scaled before running a linear discriminant analysis. The figure displays the projection of the parameter sets along the most discriminative directions. The linear discriminant analysis algorithm is from the scikit-learn python package. 


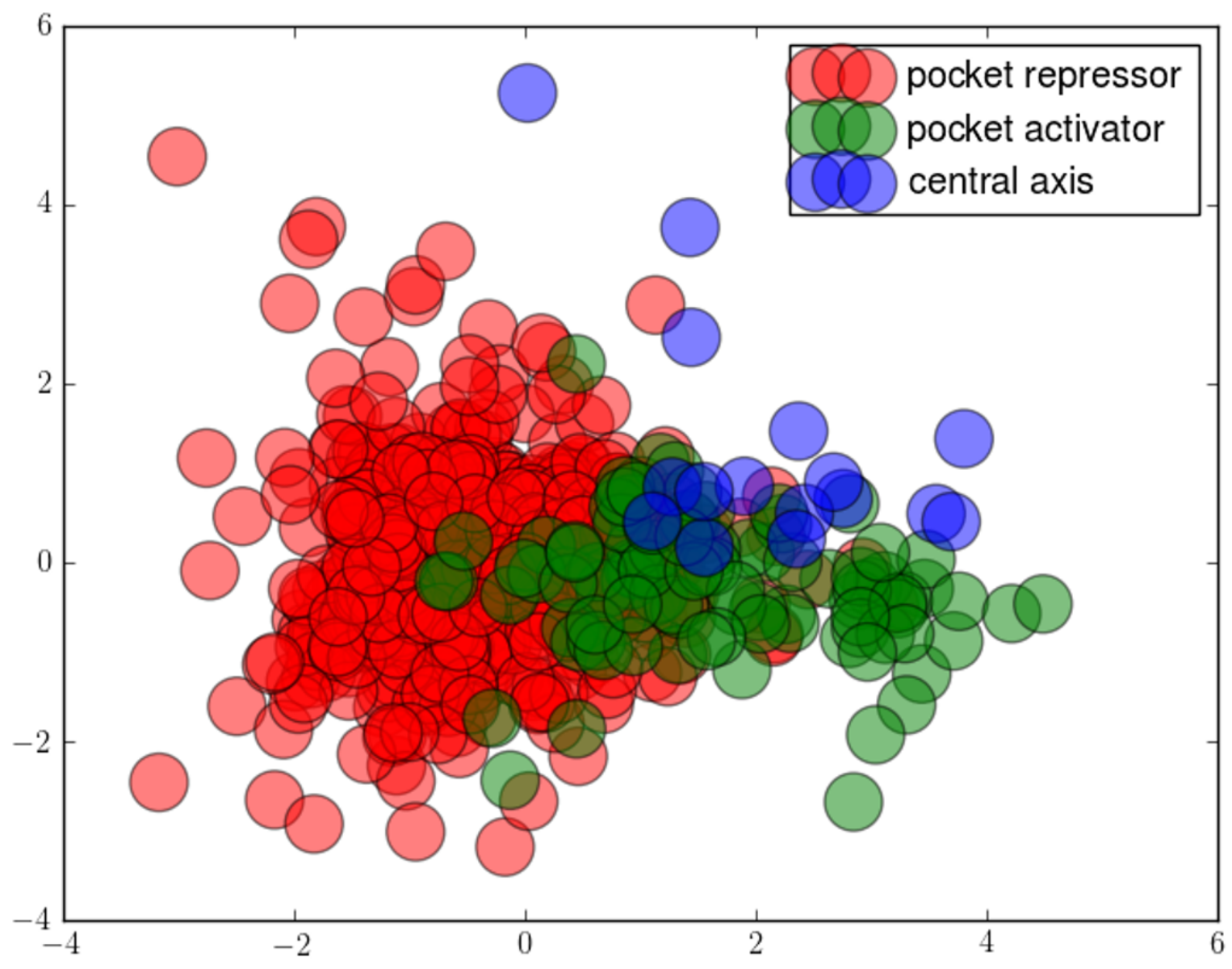

Fig S3: The three categories of behaviour can be separated in the parameter space of mobility parameters. The base 10 logarithm of mobility parameter values $\left(D_{w}, D_{h}, D_{d}, g_{w}, g_{h}, g_{d}\right)$ were centered and scaled before running a linear discriminant analysis. The figure displays the projection of the parameter sets along the most discriminative directions. The linear discriminant analysis algorithm is from the scikit-learn python package. 

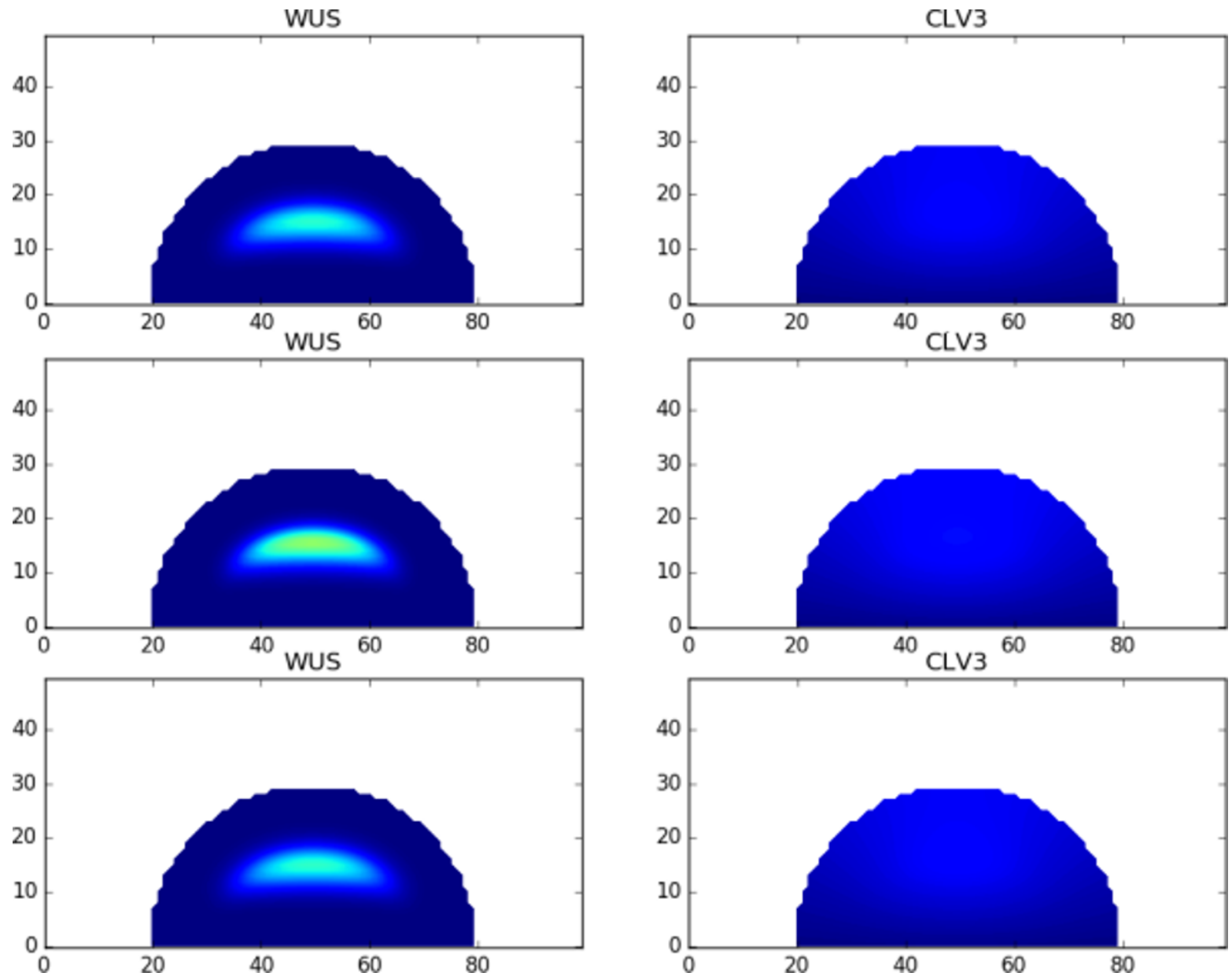

Fig S4: Optimisation results for a model based entirely on WUS regulating $C L V 3$. The optimisation procedure was adapted to test a model where WUS is the only regulator of $C L V 3$ expression. The rows show three examples of the results obtained: in this scenario the best $C L V 3$ expression is a faint hue covering the WUS expression domain and the tip of the meristem. Expression of genes in the panels goes from blue (null) to red (twice the optimisation target expression and above) via green (optimisation target). 
bioRxiv preprint doi: https://doi.org/10.1101/237933; this version posted December 21, 2017. The copyright holder for this preprint (which was not certified by peer review) is the author/funder, who has granted bioRxiv a license to display the preprint in perpetuity. It is made available under aCC-BY-NC 4.0 International license.
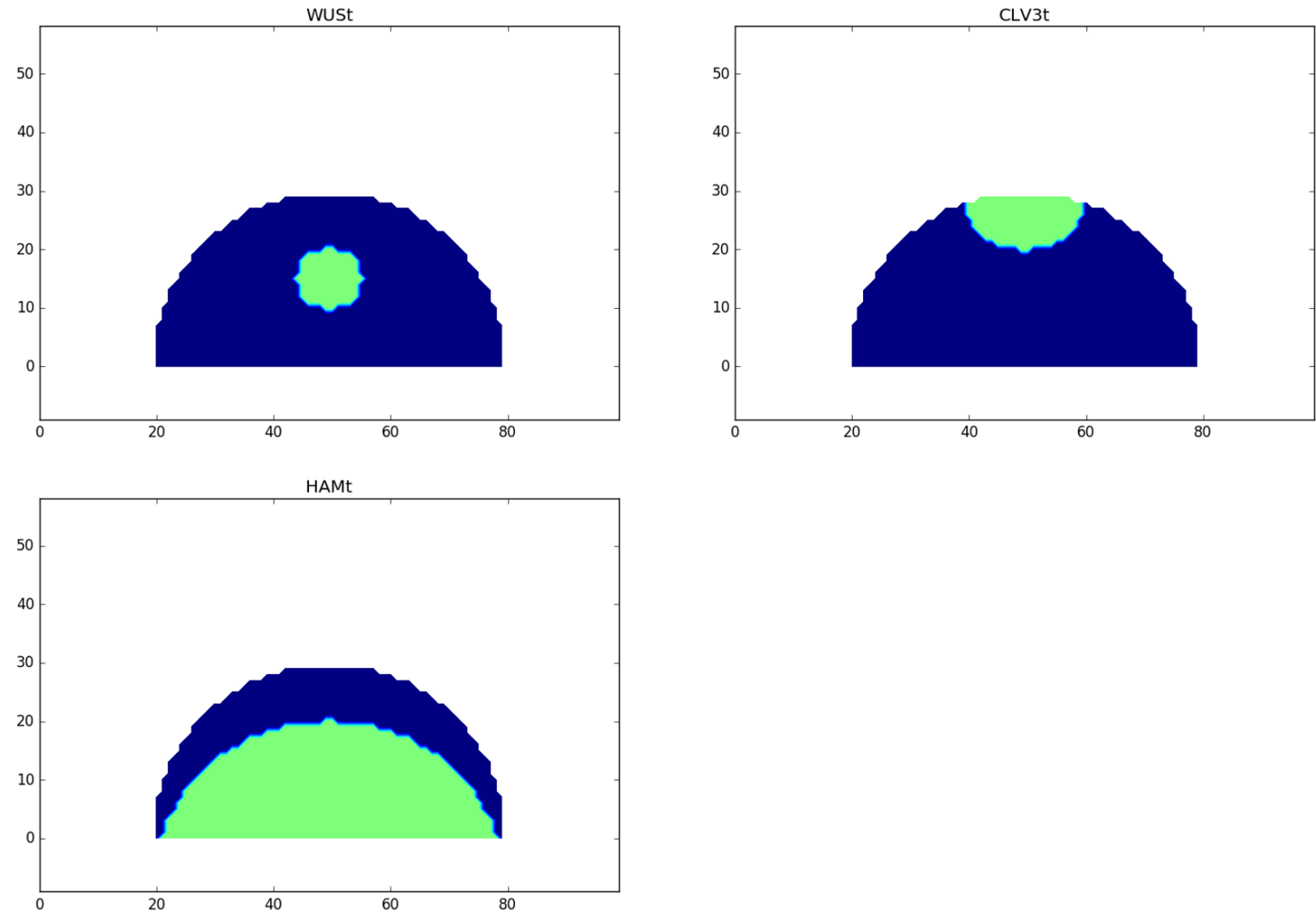

Fig S5: Optimisation target domains for WUS, CLV3 and HAM. 